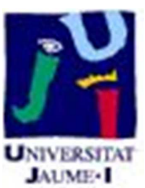

Título artículo / Títol article: Measures of Similarity Between Objects Based on Qualitative Shape Descriptions

Autores / Autors

Zoe Falomir Llansola, Luís González Abril, Lledó Museros Cabedo, Juan Antonio Ortega

Revista:

Spatial Cognition \& Computation

Versión / Versió:

Versión Pre-print

Cita bibliográfica / Cita bibliogràfica (ISO 690):

url Repositori UJI:
FALOMIR, Zoe, et al. Measures of Similarity Between Objects Based on Qualitative Shape Descriptions. Spatial Cognition \& Computation, 2013, vol. 13, no 3, p. 181-218

http://hdl.handle.net/10234/115844 


\title{
Measures of Similarity Between Objects Based on Qualitative Shape Descriptions
}

\author{
Zoe Falomir, Luis Gonzalez-Abril, \\ Lledó Museros and Juan Antonio Ortega \\ Universität Bremen, Universitat Jaume I \\ and Universidad de Sevilla
}

\begin{abstract}
A computational approach for comparing qualitative shape descriptions (QSDs) of objects within digital images is presented. First, the dissimilarity of qualitative features of shape is measured: (i) intuitively using conceptual neighbourhood diagrams; and (ii) mathematically using interval distances. Then, a similarity measure between QSDs is defined and tested using images of different categories of the MPEG-7-CE-Shape-1 library, images of tiles used to build mosaics, and a collection of Clipart images. The results obtained show the effectiveness of the similarity measure defined, which is invariant to translations, rotations and scaling, and which implicitly manages deformation of shape parts and incompleteness.
\end{abstract}

Keywords: Qualitative representation, shape, conceptual neighbourhood diagrams, interval distance, similarity measure

\section{Introduction}

Shape is probably the single most significant property that people perceive about an object. By knowing the shape, the perceiver can predict more facts about that object (what kind of object it is, what it is used for and so on) than by knowing any other property (Palmer, 1999).

Formally, the shape of an object can be described by the properties of the object's boundary. A purely quantitative representation of that boundary could be a set of mathematical functions of the coordinate space, such as a circumference is

Correspondence concerning this article should be addressed to Dr.-Ing. Zoe Falomir Llansola, Cognitive Systems Department, Universität Bremen E-mail: zfalomir@gmail.com 


\section{Z. Falomir et al.}

represented mathematically by $x^{2}+y^{2}=r^{2}$. However, the more complex the shape is, the more complicated is the numerical function that describes its boundary. In these cases, piecewise interpolation methods are typically used; i.e. the shape of the object is described by approximation using a set of small parts, such as straight lines or flat surfaces, for which numerical functions may be found. The set of all the functions represents the quantitative description of the object's shape. A widely used alternative to this method is to make a quantitative representation of the object's shape as a function of the pixels in the object image (Forsyth and Ponce, 2003). In this case, however, the result is coarser or finer depending on the resolution used, because some pixels may be only partly occupied, and it may also vary considerably if the object is rotated or in different positions in the pixel grid.

Because of the numerical properties of digital images, most of the image processing in computer vision has been carried out by applying mathematical models and other quantitative techniques to describe and identify the shape of the objects contained in an image. However, qualitative representations of a shape (i.e. based on linguistic terms such as circle, curve, acute etc.) are most useful for applications of AI that involve user communication, as Gottfried (2008) mentions:

'Interestingly, imprecise descriptions are frequently sufficient, and in particular, frequently related to the human's visual system, who can comprehend such qualitative, imprecise features better than precise quantitative measurements'

that is a human user can understand more easily what an obtuse angle is rather than what an angle of $156.74^{\circ}$ means.

Qualitative shape descriptions focus on the relevant and invariant features of shape that are considered most important within a particular context, including how many concavities a shape has, whether it has any lines of symmetry, if it is rectilinear or curvilinear, or if it has any holes, etc. (Galton and Meathrel, 1999). Taking a broader view, the origin of qualitative descriptions is probably obtaining a high level description of low level inputs. Some of the most relevant works in qualitative shape description are those by: Schlieder (1994); Cohn (1995); Damski and Gero (1996); Clementini and Di Felice (1997); Gero (1999); Galton and Meathrel (1999); Leyton (2004); Museros (2006); Gottfried (2009). All these works provide evidence for the effectiveness of using qualitative information to describe shapes (for a detailed comparison of these works with our approach see Museros et al. (2011)).

In this paper, shape description is tackled from a qualitative perspective, in which a set of values related to a feature of shape is represented with only a qualitative tag. The idea of this proposal is to abstract the numerical values, as has previously been done by Lesher et al. (2000); Lin et al. (2003) and Robinson 
(2007) using the discretisation ${ }^{1}$ of continuous values. The approach used here for qualitative shape description of silhouettes of two-dimensional objects was first outlined in Falomir et al. (2008) and Falomir et al. (2011) as an extension of the model by Museros and Escrig (2007) and Museros et al. (2011) used to describe the shape of the edges of the tiles that were automatically assembled into a ceramic mosaic by a robot arm. That earlier model was improved by Falomir et al. (2008) to reduce the ambiguity in describing all existing 2D objects (not only tiles) and then it was used in Falomir et al. (2011) to describe the shape of the objects appearing in any digital image captured by a robot camera. Here, the approach by Falomir et al. (2008) is defined in a general way that allow adaptation according to the given application and the AMEVA algorithm (González-Abril et al., 2009) is used to discretize our qualitative features of shape.

After processing the low level inputs and abstracting them to qualitative or high level descriptions, an important problem is to obtain a method that quantifies the resemblance or closeness between these descriptions. Usually these methods are based on exact matching of descriptions, which can only determine if both descriptions are equally the same or not, or approximate matching, which can obtain a degree of similarity between both descriptions although they are not equal. The approach Museros et al. (2011) applied exact matching to find a correspondence of qualitative shape descriptions. Later, the improved shape description model by Falomir et al. (2008) was tested on tiles in mosaic assembling by approximate matching using a simple definition of shape similarity based on conceptual neighbourhood diagrams (Falomir et al., 2010). Here, that earlier similarity method is improved an tested on different kinds of 2D objects (not only tiles) and an alternative method based on interval distances is presented and compared to the previous one. Therefore, an approach for shape similarity calculus is presented in this paper which determines the similarity of qualitative features of shape: (i) intuitively from conceptual neighbourhood graphs and (ii) mathematically from interval distances. Then this approach is tested on some image categories from the MPEG-7 CE Shape-1 library, images of tiles used to build mosaics and on Clipart images, showing the flexibility and adaptability of our approach. The main of our approach is to obtain a similarity degree between shapes from a cognitive perspective, that is, which approximates to the human shape similarity assessment, and analysis of our results dealing with this issue are presented through the paper.

The rest of this paper is structured as follows. Section 2 reviews the literature on shape similarity methods. In Section 3, the qualitative model used for shape description is presented. A new approach for shape similarity calculus is developed in Section 4. Section 5 presents an experimental evaluation. Finally, conclusions are drawn in Section 6.

\footnotetext{
${ }^{1}$ Discretisation is a process of transforming continuous attribute values into a finite number of intervals and associating a discrete value with each interval.
} 


\section{Z. Falomir et al.}

\section{Literature Review}

Shape similarity has been widely studied. In the literature works can be found that define similarity measures between shapes that are represented by: (i) quantitative information (Super, 2004; Ling and Jacobs, 2007; Attalla and Siy, 2005; Latecki and Lakamper, 2000; Bai et al., 2008; Gdalyahu and Weinshall, 1998; Mori et al., 2001); (ii) mixed quantitative and qualitative information (Shokoufandeh et al., 2002; Berretti et al., 2000; Siddiqi et al., 1998; Macrini et al., 2008; Sebastian et al., 2001, 2002) and (iii) qualitative information (Gottfried, 2008; Kuijpers et al., 2006; Schuldt et al., 2006).

Approaches to shape similarity calculus based on quantitative representations can be classified into:

- approaches that match points of the shape boundary: Super (2004) defines critical points of high curvature on boundaries and normalises the shape to a reference frame for rotation and scaling before calculating a distance measure used in the matching process; whereas Ling and Jacobs (2007) consider the inner-distance, or the length between landmark points within the shape silhouette to define shape descriptors invariant to articulation, which improved the classification of articulated shapes of 2D objects.

- approaches that match segments of the shape boundary: (i) shapes are segmented at multiple resolutions and a similarity is defined by elastic matching of shape segments in the work by Attalla and Siy (2005); (ii) a similarity measure between shapes based on the correspondence of visual parts where partial matching can be performed when the scale is known is presented by Latecki and Lakamper (2000) and then it is used for detection and recognition of contour parts in digital images by Bai et al. (2008); (iii) multiscale random fields are used by Latecki et al. (2008) for contour grouping and recognizing shapes when the scale is unknown; and (iv) a local curve matching algorithm is described by Gdalyahu and Weinshall (1998) that extracts points of high curvature and calculates a distance between them using efficient alignment.

- approaches that match the context of the shapes: Mori et al. (2001) define a shape feature descriptor vector that is used to represent general shape contour.

Approaches to shape similarity that mix quantitative and qualitative representations are those based on graphs/trees that usually describe the spatial arrangement of the shape parts between them but also contain some measurable properties of each shape part in their edges/nodes. For example: (i) Shokoufandeh et al. (2002) divide the coarse shape of an object into blobs and geometric relationships between them are organised into a graph, which is used for shape comparing; (ii) 
a shape is divided into tokens, according to its protrusions, and arranged into an M-tree, which is used to calculate distances between tokens and to obtain a dissimilarity measure between the M-trees of two shapes by Berretti et al. (2000); (iii) shocks (singularities) of a curve on bounding contours are organised into a graph for shape matching by Siddiqi et al. (1998) and evolve to skeletons ${ }^{2}$ and bone graphs for object recognition in the work by Macrini et al. (2008); finally, (iv) a distance between shock graphs is defined and used for recognition of shapes in the work by Sebastian et al. (2001) and for retrieval of similar shapes in large databases in the work by Sebastian et al. (2002).

The most representative approaches to shape similarity based on qualitative representation can be generally classified as:

- based on qualitative shape descriptors: (i) bipartite arrangements defined by Gottfried (2008) that relate line segments of a contour of an object to other parts of that same contour and then a similarity measure between these qualitative descriptions of shape is given; (ii) matrices of qualitative concepts developed by Kuijpers et al. (2006) using the double-cross orientation model by Freksa (1992) to describe polylines and to find a similarity measure between polygons; and finally, (iii) polygons are described qualitatively by their scope (calculated as their relative position with respect to one of their line segments where the double-cross grid described by Freksa (1992) is located) and scope histograms generated and used for shape comparing by Schuldt et al. (2006).

- theoretical approaches: (i) the recognition-by-components theory by Biederman (1987) in which any object can be generated from a set of generalizedcone components, called geons; (ii) the relational modelling technique by Shapiro et al. (1980) which decomposes objects into sticks, plates and blobs; and finally, (iii) the codons by Richards and Hoffman (1985) that are simple primitives for describing closed 2D shapes.

Finally, it is worth to mention that some invariant feature descriptors and detectors methods (such as SIFT by Lowe (2004), SURF by Bay et al. (2006), SIFT + Vocabulary Tree by Csurka et al. (2004), Harris-Affine and Hessian-Affine by Mikolajczyk et al. (2005), MSER by Matas et al. (2002), etc.), which address the problem of comparing two digital images, have been applied to object detection, where the key image contains an object and the other image is an scene with that object within it. They have become very popular because they can obtain an object hypothesis location within the scene image in a fast an scalable way by analyzing all the pixels of both raw PGM images and doing mathematical operations on them. However, they obtain false positives in images containing few textures (that

\footnotetext{
${ }^{2} \mathrm{~A}$ skeleton or axis is a two dimensional arc reflecting some global or local symmetry or regularity within a shape.
} 


\section{Z. Falomir et al.}

is, images with simple object shapes, such as web icons, Clipart images, etc.) or images containing repetitive patterns. And finally, they do not describe and compare object shapes at all and they cannot obtain a similarity index between shapes, as all the previous mentioned works do.

\section{A Qualitative Shape Description}

When people describe the shape of an object, we usually distinguish between straight sides and curved ones, describe angles and their convexity, compare the lengths of the sides of the object, etc. Hence, these features are the most relevant ones, from an intuitive point of view, for describing shapes and this is the main reason why we use them, after they were formally defined in Falomir et al. (2008). Accordingly, this approach called QSD (Qualitative Shape Description), which is based on how intuitively human beings describe shapes, is used in this paper.

Given a digital image containing a two-dimensional object, our approach for Qualitative Shape Description (QSD) automatically extracts the closed boundary of this object applying an image segmentation method (i.e. the colour segmentation method by Felzenszwalb and Huttenlocher (2004) or the well-known segmentation method by Canny (1986)).

From all the points that define the boundary $(N)$, a set of relevant points (RPSet) of the shape is extracted as described in Algorithm 1. The points of a boundary that are considered consecutive are those separated by a pre-established granularity step $(k)$. If the slope between a point $P_{i}$ and its consecutive point $P_{i+k}$,

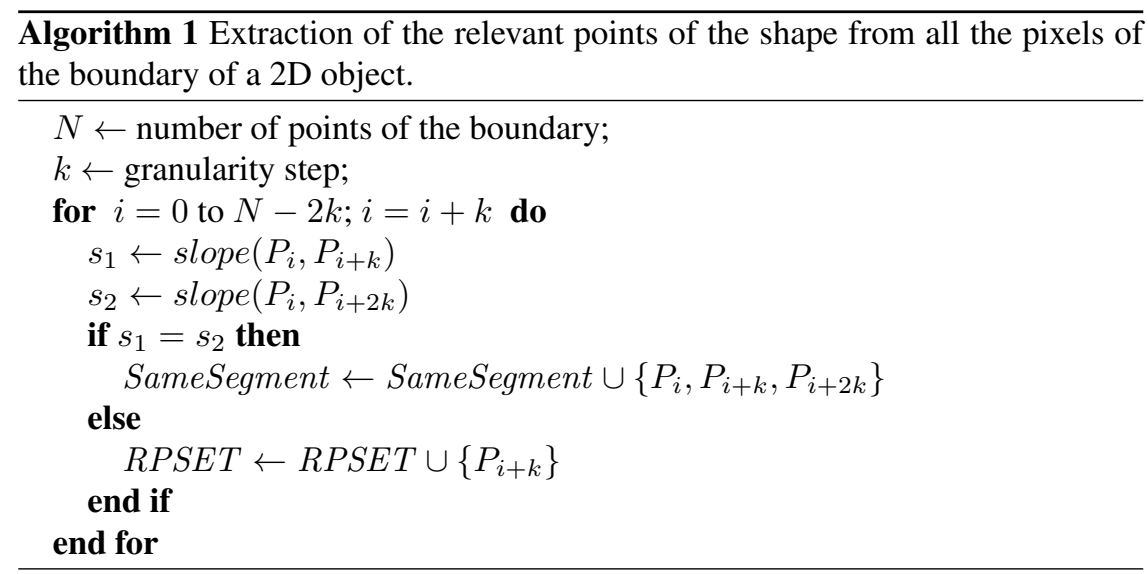

denoted by $s_{1}$, and the slope between $P_{i}$ and $P_{i+2 k}$, termed $s_{2}$, are equal, then $P_{i}$, $P_{i+k}$ and $P_{i+2 k}$ belong to the same straight segment. If $s_{1}$ and $s_{2}$ are not equal, $P_{i}, P_{i+k}$ and $P_{i+2 k}$ belong to different straight segments or to a curved segment. This process is repeated for a new point $P_{i+3 k}$ and the process stops when all the 
consecutive points of the boundary are visited. $P$ is considered a relevant point if it is the point at which the slope stops being constant or it is the point at which the slope changes its sign. Note that the granularity step is set by experimentation as a function of the edge length of the described object: if the edges are long, the granularity step will have a larger value; if they are short, the granularity step will have a smaller value.

Finally, a set of relevant points, denoted by $\left\{P_{0}, P_{1}, \ldots, P_{n}\right\}$, determines the shape of the object. Each of those relevant points $P$ is described by a set of four features, which are defined below:

$$
<\mathrm{EC}_{p}, \mathrm{~A}_{p} \mid \mathrm{TC}_{p}, \mathrm{~L}_{p}, \mathrm{C}_{p}>
$$

The first feature is the Edge Connection (denoted by EC) and it indicates the connection occurring at the relevant point $P$. This feature is described by the following tags:

- line-p-Line $(\mathrm{lpL})$, if the point $P$ connects two straight lines;

- line-p-Curve(lpC), if $P$ connects a line and a curve;

- curve-p-Line (cpL), if $P$ connects a curve and a line;

- curve-p-Curve (cpC), if $P$ connects two curves; or

- curve-p (cp) or $p$-Curve( $\mathrm{pC})$, if $P$ is a point of curvature of a curve.

If the EC is a line-p-Line, line-p-Curve, curve-p-Line or curve-p-Curve, the second feature to consider is the Angle (denoted by $\mathbf{A}$ ) at the relevant point. The angle is a quantitative feature that is discretised using the Angle Reference System or $\mathrm{ARS}=\left\{{ }^{\circ}, \mathrm{A}_{L A B}, \mathrm{~A}_{I N T}\right\}$ where, degrees $\left(^{\circ}\right)$ indicates the unit of measurement of the angles; $\mathrm{A}_{L A B}$ refers to the set of labels for the angles; and $\mathrm{A}_{I N T}$ refers to the values in degrees ${ }^{\circ}$ related to each label: $\mathrm{A}_{L A B}=\left\{\mathrm{A}_{1}, \mathrm{~A}_{2}, \ldots, \mathrm{A}_{K A}\right\}$, and $\mathrm{A}_{I N T}=\left\{\left[0, \mathrm{a}_{1}\right],\left(\mathrm{a}_{1}, \mathrm{a}_{2}\right], \ldots,\left(\mathrm{a}_{K A-1}, 180\right]\right\}$ where $K A$ is the number of labels ${ }^{3}$.

On the other hand, if the EC is a curve-p or a $p$-Curve, the second feature is the Type of Curvature (denoted by TC) at $P$ which is defined by the Type of Curvature Reference System or TCRS $=\left\{{ }^{\circ}, \mathrm{TC}_{L A B}, \mathrm{TC}_{I N T}\right\}$. As it is shown in Figure 1, the Type of Curvature at $P_{j}$ is determined by calculating first the point $c$, which is the half-point of the line between $P_{j-1}$ (initial point of the curve) and $P_{j+1}$ (final point of the curve). Next, the distance between $P_{j-1}$ and $c$, named $d a$, and the distance between $P_{j}$ and $c$, named $d b$, are calculated, and finally, the angle that determines the TC is obtained by $\operatorname{Angle}(P)=2 \cdot \operatorname{atan} 2(d a / d b) \cdot 180 / \pi$ in degrees $\left(^{\circ}\right)$. In TCRS, $\mathrm{TC}_{L A B}$ refers to the set of labels for curvature; and $\mathrm{TC}_{I N T}$ refers to the values of degrees $\left(^{\circ}\right)$ related to each label: $\mathrm{TC}_{L A B}=\left\{\mathrm{TC}_{1}, \mathrm{TC}_{2}, \ldots\right.$, $\left.\mathrm{TC}_{K T C}\right\}$, and $\mathrm{TC}_{I N T}=\left\{\left[0, \mathrm{tc}_{1}\right],\left(\mathrm{tc}_{1}, \mathrm{tc}_{2}\right], \ldots,\left(\mathrm{tc}_{K T C-1}, 180\right]\right\}$ where $K T C$ is the number of labels.

\footnotetext{
${ }^{3}$ The number of labels in this feature and in the other features must be defined in each situation as it can be seen in Section 5.1.
} 


\section{Z. Falomir et al.}

The third feature considered is the compared length (denoted by $\mathbf{L}$ ) which is defined by the Length Reference System or LRS $=\left\{\mathrm{UL}, \mathrm{L}_{L A B}, \mathrm{~L}_{I N T}\right\}$, where UL or Unit of compared Length refers to the relation between the length of the first edge and the length of the second edge connected by $P$, that is, $u l=$ (length of $1^{\text {st }}$ edge)/(length of $2^{\text {nd }}$ edge); $\mathrm{L}_{L A B}$ refers to the set of labels for compared length; and $\mathrm{L}_{I N T}$ refers to the values of UL related to each label: $\mathrm{L}_{L A B}=\left\{\mathrm{L}_{1}, \mathrm{~L}_{2}, \ldots\right.$, $\left.\mathrm{L}_{K L}\right\}$, and $\mathrm{L}_{I N T}=\left\{\left[0,1_{1}\right],\left(1_{1}, 1_{2}\right], \ldots,\left(1_{K L-1}, 1_{K L}\right]\right\}$ where $K L$ is the number of labels and $1_{K L}$ is the maximum value of the feature $L$.

The last feature to be considered is the Convexity (denoted by $\mathbf{C}$ ) at point $P$, which is obtained from the oriented line built from the previous point to the next point and by ordering the relevant points of the shape clockwise. If point $P_{j}$ is on the left of the segment defined by $\mathrm{P}_{j-1}$ and $\mathrm{P}_{j+1}$, then $P_{j}$ is convex; otherwise $P_{j}$ is concave. For example, as Figure 2 shows: $P_{j}$ is characterised as convex, whereas $P_{j+1}$ is characterised as concave. Note that mathematically $P_{j}$ cannot be within the oriented line from $P_{j-1}$ to $P_{j+1}$, otherwise it will not be a relevant point of the shape.

Therefore, the complete description of the shape of a 2D object is given from a set of qualitative features as follows:

$$
\begin{gathered}
{\left[\mathrm{EC}_{0}, \mathrm{~A}_{0} \mid \mathrm{TC}_{0}, \mathrm{~L}_{0}, \mathrm{C}_{0}\right],\left[\mathrm{EC}_{1}, \mathrm{~A}_{1} \mid \mathrm{TC}_{1}, \mathrm{~L}_{1}, \mathrm{C}_{1}\right], \ldots,\left[\mathrm{EC}_{n-1}, \mathrm{~A}_{n-1} \mid \mathrm{TC}_{n-1}\right.} \\
\left.\mathrm{L}_{n-1}, \mathrm{C}_{n-1}\right]
\end{gathered}
$$

where $\mathrm{n}$ is the total number of relevant points of the object, $\mathrm{EC}_{i}$ describes the Edge Connection that occurs at the point $\mathrm{P}_{i}, \mathrm{~A}_{i} \mid \mathrm{TC}_{i}$ describes the angle or the type of curvature at $\mathrm{P}_{i}, \mathrm{~L}_{i}$ describes the compared length of the edges connected at $\mathrm{P}_{i}$ and finally, $\mathrm{C}_{i}$ describes the convexity at $\mathrm{P}_{i}$. The first relevant point to be described (denoted by $\mathrm{P}_{0}$ ) is always the one closest to the upper-left corner of the image and the rest of the relevant points are described cyclically in a clockwise direction. An example of the general QSD of an object is shown in Table 1.

From a cognitive point of view, shape is defined in the MIT Encyclopedia of Cognitive Science by Wilson and Keil (1999) (see Shape Perception entry) as:

'An aspect of a stimulus that remains invariant despite changes in size, position and orientation.'

Therefore, it is important to note that the QSD presented here is:

- invariant to scaling (expansions and contractions in size). If a shape is scaled, then all the edges are expanded or contracted in the same proportion, and therefore the features of shape obtained in both situations are the same. If a shape is expanded or contracted until an edge disappears, then our approach considers that the original shape is transformed into another different one because they have different quantity of edges. 
- invariant to translations (changes in position), because an object description is always started at the point closest to the upper-left corner of the image, and therefore does not depend on where the object is located in the image.

Clearly, the QSD is not invariant to rotations (changing orientation), however the comparison of two shapes would be invariant to rotation if both shape descriptions were compared considering each point as the possible starting point of the (cyclic) description. This is one constraint that must be considered when defining a cognitive similarity measure between shapes described by our QSD.

\section{Similarity Between QSDs}

With the aim of defining a similarity measure between two QSDs corresponding to two objects, it is necessary to work through three stages:

- defining a similarity measure between the qualitative features (related to the shape features Edge Connection (EC), Angle (A), Type of Curvature (TC), compared Length $(\mathrm{L})$ and Convexity $(\mathrm{C})$ ) that describe the relevant points of each QSD of the objects compared (Section 4.1);

- obtaining a similarity measure between a pair of relevant points: each one corresponding to the QSD of each compared object (Section 4.2);

- defining a similarity measure between the QSDs of both objects by establishing a correspondence of pairs of relevant points (Section 4.3).

\subsection{Similarity of Qualitative Features}

In order to compare the qualitative tags defined for each feature of shape (Edge Connection (EC), Angle (A), Type of Curvature (TC), compared Length (L) and Convexity (C)), matrices of dissimilarity values are built:

- for features EC, A, TC, L and C, from a qualitative and cognitive perspective, using conceptual neighbourhood diagrams (Section 4.1.1); and

- for features A, TC and L (defined using a reference system based on intervals) from a quantitative perspective, using interval distances that are richer mathematically (Section 4.1.2).

\subsubsection{Building Dissimilarity Matrices Using Conceptual Neighbourhood Diagrams (CNDs)}

The possible transformations between two labels that describe a feature can be defined from its corresponding CND. The term conceptual neighbourhood was 


\section{Z. Falomir et al.}

first considered by Freksa (1991) in his analysis of the 13 interval relations defined in the temporal logic defined by Allen (1981):

'Two relations between pairs of events are conceptual neighbours if they can be directly transformed one into another by continuous deformation (i.e. shortening or lengthening) of the events.'

Conceptual neighbourhood relations can be found between the qualitative tags defined for each feature of shape in the QSD model. For example, when dealing with angles, the qualitative names acute and right can be considered conceptual neighbours because a quantitative extension of the angle acute leads to a direct transition to the angle right. However, angles acute and obtuse are not conceptual neighbours, because a transition between them must go through the angle right first.

In general, $C N D s$ can be described as diagrams or graphs containing: (i) nodes that map to a set of individual relations defined on regions or intervals and (ii) paths or edges connecting pairs of adjacent nodes that map to continuous transformations between them. From the CNDs defined, a dissimilarity matrix between qualitative tags represented in each CND can be calculated as the minimal path between them.

Figure 3 presents the CND for the feature Edge Connection (EC) according to a bending continuous deformation, in which the qualitative tags that are conceptual neighbours are those that represent only a change from a curve to a line or viceversa. If a line is changed for a curve and also a curve is changed for a line the dissimilarity between concepts is 2 . The dissimilarity matrix defined for this CND is shown in Table 2. Note that this dissimilarity measure corresponds to Levenshtein (1966)'s distance between the abbreviations of the qualitative tags defined for EC. Levenshtein's distance (LD) is an editing distance that measures the amount of differences between two strings. It is defined as the minimum cost (number of edits) needed to transform one string into another with the allowable operations: insertion, deletion or substitution of a single character. For instance, in the case of EC abbreviations, the LD between $l p L$ and $c p$ is 2 , because 1 substitution and 1 insertion are needed, the LD between $c p C$ and $l p C$ is 1 because 1 substitution operation is needed, whereas the LD between $l p L$ and $c p C$ is 2 because two substitution operations are needed.

The CND for the feature Convexity (C) according to a smashing continuous deformation is shown in Figure 4 and the dissimilarity matrix defined for this CND is shown in Table 3.

As the features of shape Angle (A), Type of Curvature (TC) and compared Length (L) are defined on continuous intervals of values, the general CNDs for them according to a shortening or lengthening continuous deformation correspond to those shown in Figures 5, 6 and 7. Their corresponding dissimilarity matrices are calculated in general from (1), (2) and (3) based on the minimal path between 
the nodes of the CND.

$$
\begin{gathered}
d\left(A_{i}, A_{j}\right)=|i-j| \text { where } i, j=1 \ldots K_{A} \\
d\left(T C_{i}, T C_{j}\right)=|i-j| \text { where } i, j=1 \ldots K_{T C} \\
d\left(L_{i}, L_{j}\right)=|i-j| \text { where } i, j=1 \ldots K_{L}
\end{gathered}
$$

Finally, it is our belief that employing CNDs in this way is plausible and intuitive.

\subsubsection{Building Dissimilarity Matrices Using Interval Distances}

To define the dissimilarity matrices for the features angle (A), type of curvature (TC) and compared length (L), an ordinal scale has been used in Section 4.1.1. However, these features are defined from intervals of values in their Reference Systems. Therefore, we can take advantage of this by not considering dissimilarity matrices but instead, distance matrices, which are richer mathematically, because the concept of distance is stricter than the concept of dissimilarity.

Let us introduce the concept of interval distance. Given an open interval (analogously for another kind of interval) of finite dimension, there are two main ways to represent it: from the extreme points as $(a, b)$ (classical notation) or as an open ball $\mathrm{B}_{r}(\mathrm{c})$ (Borelian notation) where $c=(a+b) / 2$ (centre) and $r=(b-a) / 2$ (radius). Given two intervals, $I_{1}=\left(a_{1}, b_{1}\right)=B_{r 1}\left(c_{1}\right)$ and $I_{2}=\left(a_{2}, b_{2}\right)=B_{r 2}\left(c_{2}\right)$, a family of distances between intervals was defined by Gonzalez-Abril et al. (2004), which depends on three parameters as follows:

$$
d^{2}\left(I_{1}, I_{2}\right)=(\Delta c \Delta r) A\left(\begin{array}{c}
\Delta c \\
\Delta r
\end{array}\right)
$$

where $\Delta c=c_{2}-c_{1}, \Delta r=r_{2}-r_{1}$ and $A$ is a symmetrical $2 \times 2$ matrix of weights, which must be a positive definite matrix. From the $A$ matrix, the weights given to the position of the intervals and to the radius can be controlled.

In this paper we will use the most natural choice for the $A$ matrix, which is the identity matrix that provides the next distance:

$$
d^{2}\left(I_{1}, I_{2}\right)=\sqrt{\Delta^{2} c+\Delta^{2} r}=\sqrt{\left(c_{2}-c_{1}\right)^{2}+\left(r_{2}-r_{1}\right)^{2}}
$$

Hence, new dissimilarity matrices can be built for the features angle, type of curvature and length considering interval distances.

\subsection{A Similarity Between Relevant Points}

As previously mentioned, the shape of an object is qualitatively described by means of all its relevant points. Therefore, in order to define a similarity measure between shapes, first a similarity measure between relevant points must be 


\section{Z. Falomir et al.}

obtained. Hence, given two relevant points, denoted by $R P_{A}$ and $R P_{B}$, belonging to the shapes of the objects $A$ and $B$ respectively, a similarity measure between them, denoted by $\operatorname{Sim}\left(R P_{A}, R P_{B}\right)$, is defined as:

$$
\operatorname{Sim}\left(R P_{A}, R P_{B}\right)=1-\sum_{i \in\{E C, A, T C, C, L\}} w_{i} \frac{d s(i)}{D s(i)}
$$

where $d s$ (feature) denotes the dissimilarity between relevant points with respect to the feature obtained from the dissimilarity matrix previously defined. Ds (feature) denotes the maximum dissimilarity in the dissimilarity matrix related to the feature considered at the relevant point. Hence, by dividing $d s$ (feature) and $D s$ (feature) the proportion of dissimilarity related to feature of $\mathrm{RP}_{A}$ and $\mathrm{RP}_{B}$ is obtained, which is between 0 and 1 . Moreover, the parameter $w_{\text {feature }}$ is the weight assigned to this feature, and it holds that $w_{E C}+w_{A}+w_{L}+w_{C}=1, w_{A}=w_{T C}$ and $w_{\text {feature }} \geq 0$ for each feature.

In this paper, with the aim of giving the same importance to all features in (6), all the weights have the same value: $\frac{1}{4}$. Clearly, these weights can be tuned if a researcher needs to give more importance to one feature over the others. Furthermore, in (6) the dissimilarity value is subtracted from 1 with the aim of providing a similarity measure.

Hence, $0 \leq \operatorname{Sim}\left(R P_{A}, R P_{B}\right)=\operatorname{Sim}\left(R P_{B}, R P_{A}\right) \leq 1$ and for each $R P_{A}$ and $R P_{B}$. Furthermore, if $\operatorname{Sim}\left(R P_{A}, R P_{B}\right)=0$ this means that $d s$ (feature $)=$ $D s$ (feature), that is, both relevant points have the maximum dissimilarity for all features and thus, both relevant points are as different as possible.

On the other hand, if $\operatorname{Sim}\left(R P_{A}, R P_{B}\right)=1$, then this means that ds (feature $)=$ 0 for all the features of the relevant points, and hence, these two relevant points have the same QSD. In this case, both relevant points are considered equivalent (a relation of equivalence is established between them).

If one relevant point is a point of curvature ( $c p$ or $p C$ in $\mathrm{EC}$ ) and the other compared relevant point is not, the type of curvature (feature TC) of the first relevant point will be compared to the angle (feature A) of the second relevant point. For instance, in Table 1, if the relevant point $\mathrm{A}$ of the shape is compared to the relevant point B2, the corresponding Angle at A $(\operatorname{Angle}(A))$ will be compared with the corresponding type of curvature (TC) at B2 $(T C(B 2))$. However, this is not a problem for our approach because it can compare angles with types of curvature because both features correspond to the same concept, that is, the angular amplitude at the relevant point, and both can be defined by the same values in degrees.

In Section 5.1, the chosen parameters for each feature of shape are given and all these concepts are exemplified. 


\subsection{Similarity Between QSDs}

In order to compare two shapes A and B whose QSDs have the same number of relevant points (denoted by $n$ ), the similarity between $\mathrm{A}$ and $\mathrm{B}$ is calculated from (6) as an arithmetic mean of the similarity between relevant points of both shapes cyclically in a clockwise direction. Thus, the calculation of the similarity can start each time at a different relevant point of any of the shapes. When all the possible similarities between relevant points are obtained, the similarity between A and B is the highest value of all of them.

Let us clarify this similarity calculus with an example. Let $\mathrm{T} 1$ and $\mathrm{T} 2$ be two triangles, with QSDs given by $\left\{R P_{T 1}(0), R P_{T 1}(1), R P_{T 1}(2)\right\}$ and $\left\{R P_{T 2}(0)\right.$, $\left.R P_{T 2}(1), R P_{T 2}(2)\right\}$ respectively. In this case, three similarities can be considered (for simplifying, we denote $\operatorname{Sim}\left(R P_{T 1}(i), R P_{T 2}(j)\right)$ as $\operatorname{Sim}(i, j)$ ):

$$
\begin{aligned}
& \operatorname{Sim}_{1}(T 1, T 2)=\frac{1}{3}(\operatorname{Sim}(0,0)+\operatorname{Sim}(1,1)+\operatorname{Sim}(2,2)) \\
& \operatorname{Sim}_{2}(T 1, T 2)=\frac{1}{3}(\operatorname{Sim}(1,0)+\operatorname{Sim}(2,1)+\operatorname{Sim}(0,2)) \\
& \operatorname{Sim}_{3}(T 1, T 2)=\frac{1}{3}(\operatorname{Sim}(2,0)+\operatorname{Sim}(0,1)+\operatorname{Sim}(1,2))
\end{aligned}
$$

and, the final similarity between both triangles will be the maximum of these three.

It is important to note that this final similarity provides us with a correspondence between relevant points of two shapes that will be useful further on. Thus, for instance, if the final similarity between the triangle $\mathrm{T} 1$ and $\mathrm{T} 2$ is given from the $\operatorname{Sim}_{2}(\mathrm{~T} 1, \mathrm{~T} 2)$, then the correspondence obtained is:

$$
R P_{T 1}(1) \rightarrow R P_{T 2}(0), R P_{T 1}(2) \rightarrow R P_{T 2}(1), R P_{T 1}(0) \rightarrow R P_{T 2}(2)
$$

On the other hand, if two shapes A and B whose QSDs have a different number of relevant points are compared, then there are some relevant points of one shape with no corresponding points in the other shape. In this case, the points with no corresponding pairs in the other shape are compared with a new relevant point, the void point, and the similarity between both points is zero.

Let us suppose that the number of relevant points of the shapes A and B are $n$ and $m$ respectively, and without loss of generality that $n \geq m$. In this case, $n-m$ relevant points of A are compared with the void point, and the rest are compared with the relevant points of $\mathrm{B}$ in the same way as in the previous case.

Figure 8 shows two objects, $\mathrm{A}$ and $\mathrm{B}$, with 4 and 5 relevant points respectively. When comparing A and B all the possible correspondences between the relevant points of these two objects are the following:

$$
\begin{aligned}
& \{(0, \text { void }),(1,0),(2,1),(3,2),(4,3)\},\{(0, \text { void }),(1,1),(2,2),(3,3),(4,0)\} \\
& \{(0, \text { void }),(1,2),(2,3),(3,0),(4,1)\},\{(0, \text { void }),(1,3),(2,0),(3,1),(4,2)\} \\
& \{(0,0),(1, \text { void }),(2,1),(3,2),(4,3)\},\{(0,1),(1, \text { void }),(2,2),(3,3),(4,0)\} \\
& \{(0,2),(1, \text { void }),(2,3),(3,0),(4,1)\},\{(0,3),(1, \text { void }),(2,0),(3,1),(4,2)\} \\
& \{(\mathbf{0 , 0}),(\mathbf{1}, \mathbf{1}),(\mathbf{2}, \text { void }),(\mathbf{3}, \mathbf{2}),(\mathbf{4}, \mathbf{3})\},\{(0,1),(1,2),(2, \text { void }),(3,3),(4,0)\}
\end{aligned}
$$




\section{Z. Falomir et al.}

$$
\begin{aligned}
& \{(0,2),(1,3),(2, \text { void }),(3,0),(4,1)\},\{(0,3),(1,0),(2, \text { void }),(3,1),(4,2)\} \\
& \{(0,0),(1,1),(2,2),(3, \text { void }),(4,3)\},\{(0,1),(1,2),(2,3),(3, \text { void }),(4,0)\} \\
& \{(0,2),(1,3),(2,0),(3, \text { void }),(4,1)\},\{(0,3),(1,0),(2,1),(3, \text { void }),(4,2)\} \\
& \{(0,0),(1,1),(2,2),(3,3),(4, \text { void })\},\{(0,1),(1,2),(2,3),(3,0),(4, \text { void })\} \\
& \{(0,2),(1,3),(2,0),(3,1),(4, \text { void })\},\{(0,3),(1,0),(2,1),(3,2),(4, \text { void })\}
\end{aligned}
$$

For the objects in Figure $8, \operatorname{Sim}(A, B)$ is given from the correspondence $\{(0,0)$, $(1,1),(2$, void $),(3,2),(4,3)\}$. Therefore, our approach provides additional information about the shape: RP 2 in object $\mathrm{B}$ has no corresponding RP in object $\mathrm{A}$.

Thus, the similarity for each one of all possible correspondences between the relevant points of $\mathrm{A}$ and $\mathrm{B}$ by considering the void point is obtained as:

$$
\operatorname{Sim}_{\sigma}(A, B)=\frac{1}{n} \sum_{i=1}^{m} \operatorname{Sim}\left(R P_{A} \sigma(i), R P_{B}(i)\right)
$$

where $\sigma$ denotes a cyclic correspondence of the relevant point of object A and the relevant point of object $B$.

Note that only $m$ similarities between relevant points must be considered because the similarity between a relevant point of $\mathrm{A}$ and the void point is always zero. From here, the final similarity between the shapes A and B, called $\operatorname{Sim} Q S D(A, B)$, is the maximum value of these similarities, that is,

$$
\operatorname{SimQSD}(A, B)=\max _{\sigma \in C}\left(\operatorname{Sim}_{\sigma}(A, B)\right)
$$

where $C$ denotes the set of all possible correspondences between relevant points of $\mathrm{A}$ and $\mathrm{B}$.

In Algorithm 2, the process followed to calculate the similarity value between two qualitative shape descriptions (QSD) is described in pseudocode.

The main properties of this final similarity are:

- Symmetry: $\operatorname{Sim} Q S D(A, B)=\operatorname{Sim} Q S D(B, A)$.

- Invariance to rotation, translation and scale transformations;

- Upper and lower bounds, that is,

$$
0 \leq \operatorname{SimQSD}(A, B) \leq \frac{m}{n} \leq 1
$$

for any shapes A and B, because the difference between the number of relevant points of shapes penalises the final similarity.

At this point, note that, according to Wilson and Keil (1999), our approach describes and compares shapes in a cognitive way as it takes into account most of the conditions under which people perceive two distinct objects as having the same shape, that is, invariance to changes in size, position and orientation.

In terms of the computational cost (CC), two cases can be distinguished: 


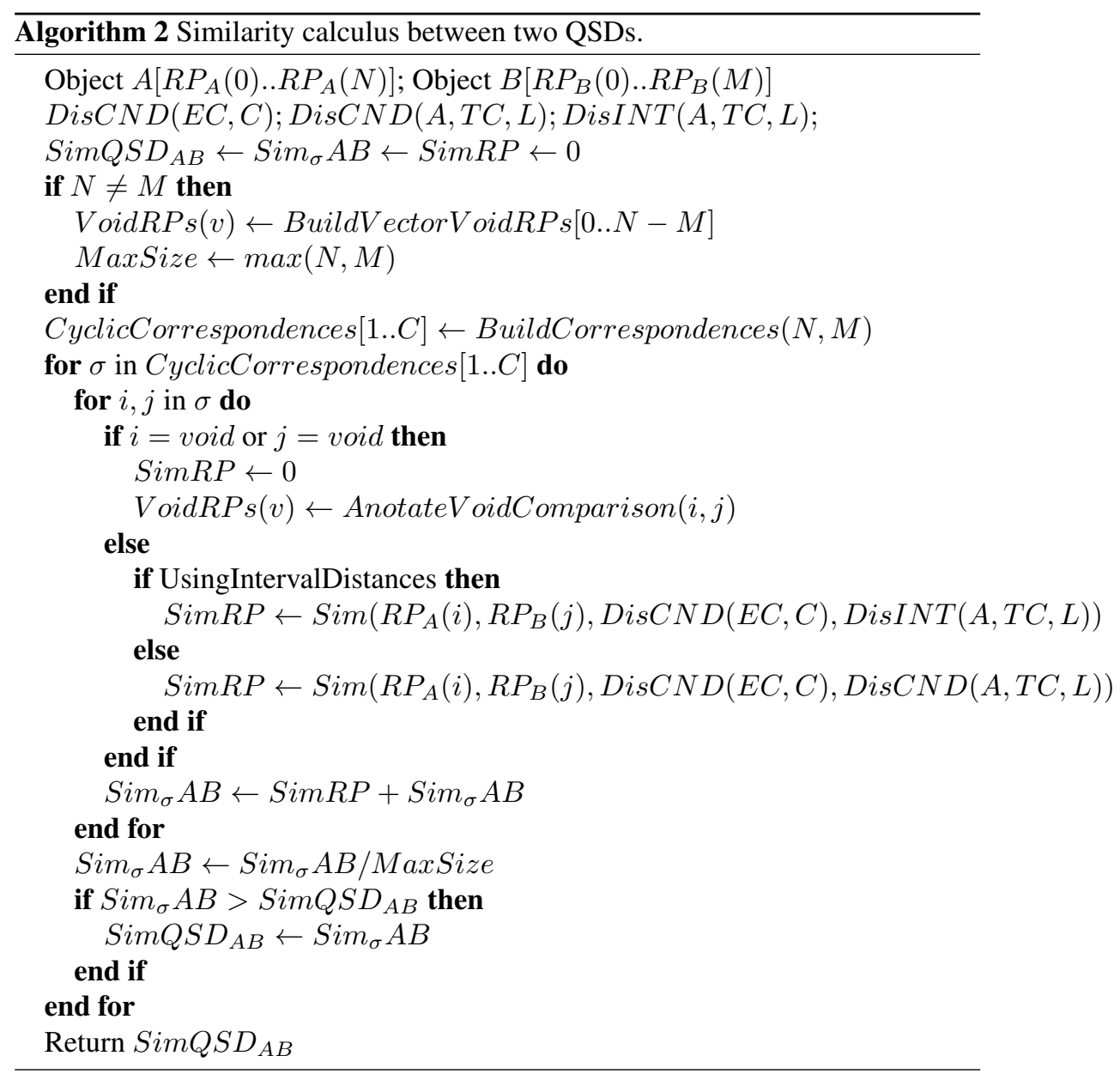

- if both shapes have the same number of relevant points $(n)$, the cost of the algorithm is $O\left(n^{2}\right)$, because the starting point of the comparison can be any point of the second shape.

- if the difference in the number of relevant points between both shapes is $n-m$, the number of possibilities for choosing $n-m$ points to be compared with the void point is a simple combinatory number

$$
\left(\begin{array}{c}
n \\
n-m
\end{array}\right)=\left(\begin{array}{c}
n \\
m
\end{array}\right)
$$

and considering that the starting point of the comparison can be any point of the shape with the highest number of relevant points, the possible cost is 


$$
\begin{aligned}
& \left(\begin{array}{c}
n \\
m
\end{array}\right) m, \text { which can be calculated to two different ways, that is, } \\
& \left(\begin{array}{c}
n \\
m
\end{array}\right) m=\frac{n !}{(n-m) ! m !} m=\frac{n(n-1) \cdots(m+1)}{(n-m) !} m=O\left(n^{n-m+1}\right) \\
& \left(\begin{array}{c}
n \\
m
\end{array}\right) m=\frac{n !}{(n-m) ! m !} m=\frac{n(n-1) \cdots(n-m+1)}{m !} m=O\left(n^{m+1}\right)
\end{aligned}
$$

and, therefore, the final cost is:

$$
\begin{aligned}
& C=\min \left\{O\left(n^{n-m+1}\right), O\left(n^{m+1}\right)\right\}=O\left(n^{\min \{m, n-m\}+1}\right) \leq O\left(n^{n / 2+1}\right) \\
& \text { since } \min \{m, n-m\} \leq n / 2 .
\end{aligned}
$$

Clearly, the computational cost (CC) peaks when two shapes with a high number of relevant points are compared and one of them has twice the number of relevant points than the other.

However, as this approach is focused on finding a similarity relation between two shapes, not a dissimilarity one, comparisons between shapes with a high difference in relevant points can be assigned a low similarity a priori and the calculation of the exact similarity value can be avoided.

Finally, note that our approach obtains all the correspondences of the relevant points and extracts those points that are compared to the void point, which detects the location of the difference in the shape. The cost of obtaining these correspondences is high and this is the reason why our approach obtains a high computational cost. This is its advantage with respect to other polynomial-time approaches (Gottfried, 2008; Latecki and Lakamper, 2000; Bai et al., 2008; Latecki et al., 2008) which have some cognitive motivation, but which are not able to obtain such explanatory information among the shapes compared.

\section{Experimentation and Results}

In our experimentation, first the features composing our QSD approach are parameterized by experts as described in Section 5.1. Then the $\operatorname{SimQSD}$ is used on some images of the Bone category extracted from the MPEG-7 CE Shape-1 library $^{4}$ (Latecki et al., 2000) and the similarity values assigned from CNDs or interval distances and the correspondences of relevant points obtained are presented in Section 5.2. Moreover, the SimQSD approach is used to compare the shape of objects from: (i) all the categories of the MPEG-7 CE Shape-1 library (Section 5.3), (ii) images of tiles used to build mosaics (Section 5.4), and (iii) Clipart images (Section 5.5). Finally, an analysis of the general results of our experimentation is given in Section 5.6.

\footnotetext{
${ }^{4}$ http://www.dabi.temple.edu/ shape/MPEG7/dataset.html
} 


\subsection{Parameters Selection}

In order to determine the qualitative tags defined for describing the Angles (A), Types of Curvature (TC) and compared Lengths (L) of a shape and its corresponding interval values, some experts in the implementation area were consulted. First, they were shown some examples of tiles and they were asked to name the corresponding items of each category: angles, types of curvature and lengths. After the sampling objects finished, the number of items/classes selected for each category was that used most by the experts. Then, each tag was related with the real value in degrees and the AMEVA algorithm (González-Abril et al., 2009) was applied. This method discretizes by maximizing the contingency coefficient matrix and therefore it is robust to noisy data, as it is proved by González-Abril et al. (2009). Finally, the AMEVA algorithm provided the classes of the intervals, which were rounded afterwards in order to obtain the same sets of intervals used in the final application.

For the Angle Reference System or ARS $=\left\{{ }^{\circ}, \mathrm{A}_{L A B}, \mathrm{~A}_{I N T}\right\}$, the chosen granularity was 5 and the set of labels for the qualitative angles and the values of degrees $\left({ }^{\circ}\right)$ related to each label were:

$\mathrm{A}_{L A B}=\{$ very_acute, acute, right, obtuse, very_obtuse $\}$

$\mathrm{A}_{I N T}=\{[0,40],(40,85],(85,95],(95,140],(140,180]\}$

and in Borelian notation these are:

$\mathrm{A}_{B r(c)}=\left\{\mathrm{B}_{20}(20), \mathrm{B}_{22.5}(62.5), \mathrm{B}_{5}(90), \mathrm{B}_{22.5}(117.5), \mathrm{B}_{20}(160)\right\}$

For the Type of Curvature Reference System or TCRS $=\left\{{ }^{\circ}, \mathrm{TC}_{L A B}, \mathrm{TC}_{I N T}\right\}$, the chosen granularity was also 5 and the set of labels for the type of curvature and the values of degrees $\left({ }^{\circ}\right)$ related to each label were:

$\mathrm{TC}_{L A B}=\{$ very_acute, acute, semicircular, plane, very_plane $\}$

$\mathrm{TC}_{I N T}=\{[0,40],(40,85],(85,95],(95,140),[140,180]\}$

and in Borelian notation these are:

$\mathrm{TC}_{B r(c)}=\left\{\mathrm{B}_{20}(20), \mathrm{B}_{22.5}(62.5), \mathrm{B}_{5}(90), \mathrm{B}_{22.5}(117.5), \mathrm{B}_{20}(160)\right\}$

For the Length Reference System or LRS $=\left\{\mathrm{UL}, \mathrm{L}_{L A B}, \mathrm{~L}_{I N T}\right\}$, the chosen granularity was 7 , the set of labels related to compared length and the values related to each label were:

$\mathrm{L}_{L A B}=\{$ much_shorter $(\mathrm{msh})$, half_length $(\mathrm{hl})$, a_bit_shorter $($ absh $)$, similar_length $(\mathrm{sl})$, a_bit_longer $(\mathrm{abl})$, double_length $(\mathrm{dl})$, much_longer $(\mathrm{ml})\}$

$\mathrm{L}_{I N T}=\{(0,0.4],(0.4,0.6],(0.6,0.9],(0.9,1.1],(1.1,1.9],(1.9,2.1],(2.1,10]\}$

and in Borelian notation these are:

$\mathrm{L}_{B r(c)}=\left\{\mathrm{B}_{0.2}(0.2), \mathrm{B}_{0.1}(0.5), \mathrm{B}_{0.15}(0.75), \mathrm{B}_{0.1}(1.0), \mathrm{B}_{0.4}(1.5), \mathrm{B}_{0.1}(2.0)\right.$, $\left.\mathrm{B}_{3.95}(6.05)\right\}$

After parameterizing the features of shape Angle (A), Type of Curvature (TC) and Length (L), an example is shown in Table 1. 


\section{Z. Falomir et al.}

The considered labels for each feature of shape were tested in experimental studies carried out in our labs that described: (i) the shape of the edges of the tiles that were automatically assembled into a ceramic mosaic by a robot arm (Falomir et al., 2010) and (ii) the shape of all the relevant colour regions of any digital image captured by a mobile robot webcam (Falomir et al., 2011).

In accordance with this parameter selection, the CNDs obtained for the features of shape Angle (A), Type of Curvature (TC) and compared Length (L) are shown in Figures 9, 10 and 11 and the corresponding dissimilarity matrices are obtained from (1), (2) and (3).

Moreover, the distance (dissimilarity) matrices for the features of shape Angle (A), Type of Curvature (TC) and compared Length (L) obtained when applying the interval distances are shown in Tables 4 and 5. Note that, although the features Type of Curvature (TC) and Angle (A) contain different qualitative concepts, their dissimilarity matrices have been defined in exactly the same way because the units and intervals are the same.

Considering the dissimilarity built from the CND for the feature Angle (calculated from (1)), the dissimilarity between the interval $[0,40]$ (corresponding to very-acute) and the interval $(40,85]$ (corresponding to acute) is 1 ; and the dissimilarity between the interval $[0,40]$ and the interval $(85,95]$ (corresponding to right) is 2. Mathematically speaking, it is not accurate to say that the second dissimilarity is double the first dissimilarity because proportional values cannot be calculated on an ordinal scale. Nevertheless, considering the dissimilarity matrix built from interval distances for the feature Angle (Table 4), the distance between the interval $[0,40]$ and the interval $(40,85]$ is 42.6 ; and the distance between the interval $[0,40]$ and the interval $(85,95]$ is 71.6 . Hence, using the proportional scale, it is absolutely accurate to compare both distances from their ratio (71.6/42.6), obtaining that one is 1.7 times the other, which can be rounded up to 2 (the same dissimilarity obtained from CNDs, which proves its suitability).

Furthermore, the value $\frac{d s(i)}{D s(i)}$ in (6) can be seen as the importance of changes in each feature of shape. Hence, from the dissimilarity matrices obtained from CNDs, the following maximums $(D s(i))$ are obtained: for convexity $(\mathrm{C}), 1$; for edge connection (EC), 2; for angle (A) and type of curvature (TC), 4; and for length $(\mathrm{L}), 6$. As the value assigned to each change is 1 , this means that each change in each feature has a different importance (I) in (6) and the following priorities among features are given:

$$
I(C)=1>I(E C)=\frac{1}{2}>I(A)=I(T C)=\frac{1}{4}>I(L)=\frac{1}{6} .
$$

For the interval distance matrices, the maximums $(D s(i))$ for each feature are: for angle (A) and type of curvature (TC), 140; and for length (L), 6.95. The mean value of change between the qualitative concepts of the distance matrix for angle and type of curvature is approximately 35 for each line and row (as the 
dissimilarity matrix is symmetric), which gives us an importance of change of $35 / 140$ or $1 / 4$. Moreover, the value of change between the tags of the distance matrix for Length is approximately 1.18 for each line and row, which gives us an importance of change in the feature of $1.18 / 6.95$ or $17 / 100$. Hence, it is calculated that

$$
I(C)=1>I(E C)=\frac{1}{2}>I(A)=I(T C)=\frac{1}{4}>I(L)=\frac{17}{100} .
$$

Therefore, the priorities given when considering dissimilarities from matrices built from CNDs or from interval distances have the same order and approximately equal values of importance.

These priorities can be justified as being suitable for comparing shapes intuitively. In Figure 12 five shapes are shown $(S 1, S 2, S 3, S 4$ and $S 5)$ that exemplify these priorities. The Convexity $(C)$ is the feature that has the greatest priority because, when it changes, not only the boundary of the object changes, but also its interior (i.e. compare shapes $S 1$ to $S 2$ in which only the convexity of relevant point 2 changes). The Edge Connection (EC) is the second most important feature because it differentiates between curves and straight lines, which is also an important difference. For example, if we compare shapes $S 1$ to $S 3$ in which only the EC of relevant point 2 changes, we will see that they are more similar than $S 1$ and $S 2$ and than $S 2$ and $S 3$ in which both the EC and the C of 2 is different. The next most important feature is the angle or type of curvature because it characterises the shape of an object in a more significant way than the lengths of the edges, which usually depend on the angle they define. If we compare $S 3$ and $S 4$, the most perceptible difference is that the Angle of 2 is different, but the compared length between relevant points 3-4 and 4-0 is also different in both shapes and this is less perceptible. Finally, note that it is also true that the more similar the number of relevant points between shapes, the higher the similarity, since $S 1-S 4$ are more similar to each other than any of them are to $S 5$, which has a relevant point less than them.

\subsection{Similarity Values and Correspondences of Points Between Shapes of the Bone Category of MPEG-7 Library}

In this section, the $\operatorname{Sim} Q S D$ is tested on the images of the Bone category extracted from the MPEG-7 CE Shape-1 library. This category was selected because it has images with interesting aspects to study (deformations, incompleteness and large differences in the quantity of relevant points) that enable us to test the suitability of the obtained correspondence of relevant points between shapes.

Figure 13 shows that this approach intuitively detects the "extra" relevant points of a shape. Given the shapes Bone-1 and Bone-7, the calculation of the SimQSD provides the following results: 
- The $\operatorname{Sim} Q S D$ is started at relevant point 1 of Bone- 1 and at relevant point 0 of Bone-7, which correspond to one another;

- The relevant points of Bone-7 with no correspondence in Bone-1 are 6 and 16 , which are easily identified to the human vision; and,

- The SimQSD between shapes is 0.88 using CNDs and 0.9 using interval distances. A high similarity is obtained by both methods because Bone-7 is exactly the same as Bone- 1 with a bend in it.

Some other shapes extracted from the MPEG-7 CE Shape-1 library (see Figure 13) have been used to calculate the $\operatorname{Sim} Q S D$ between all shapes and to study the obtained correspondence of relevant points. First, using dissimilarity matrices built from CNDs (see Table 6) and secondly, using dissimilarity matrices built from CNDs for the features edge connection (EC) and Convexity and using dissimilarity matrices built from interval distances for the features of angle (A), type of curvature (TC) and length (L) (see Table 7).

In Tables 6 and 7, each cell indicates the SimQSD between the shapes, the starting points of the similarity calculus and the relevant points without correspondence. For example, in Table 6 the SimQSD between "Bone-1 and Bone-6" is 0.88 , starting the comparison by point 0 of Bone- 1 and point 15 of Bone- 6 (note that both points have the same location in the images) obtaining that relevant points 10 and 11 of Bone-1 (the shape with the greatest number of relevant points) are compared to the void point, that is they have no correspondence with relevant points in Bone-6. In Table 7 the SimQSD between "Bone-1 and Bone6 " is 0.89 , starting the comparison by point 0 of Bone- 1 and point 8 of Bone- 6 (another possible alternative) obtaining that relevant points 1 and 2 of Bone- 1 are compared to the void point.

It can be noticed in Tables 6 and 7 that the similarities obtained using CNDs or interval distances are very similar. Only a few of the similarity values calculated (marked in bold) are different. Therefore, from now on, in the paper only the results of our approach using CNDs and interval distances will be shown because the weights assigned to the CNDs can be considered a particular case of the interval distances obtained.

Finally, it is important to note that our approach obtains a high similarity value between nearly symmetrical shapes (such as Bone-7 and Bone-8) and it also tackles the problem of deformations and incomplete shapes implicitly. Bone-18 can be considered as Bone-1, but incomplete, because the top of the bone that appears in all the other images does not appear in Bone-18. Furthermore, Bone-7, Bone- 8 and Bone- 17 can be considered as Bone-1, but broken or deformed in the middle. Moreover, the relevant points of one shape that do not have a correspondence in the other shape show where the deformation or the incomplete part of the other shape is. Therefore, our approach can obtain suitable similarities between instan- 
ces of the same shape that are deformed or incomplete and give an approximate location of such deformation or incompleteness.

\subsection{Similarity Values Between Different Shape Categories of MPEG-7 Library}

In this section, the bulls-eye test ${ }^{5}$ for shape retrieval was performed using the SimQSD on the images of the MPEG-7 CE Shape-1 library. In this test, every shape in the database is compared to all other shapes, and the number of shapes from the same class among the 40 most similar shapes is reported. The bull's eye retrieval rate is the ratio of the total number of shapes from the same class to the highest possible number. Thus, the best possible rate is $100 \%$. For image segmentation and closed contour extraction, the method by Felzenszwalb and Huttenlocher (2004) was used.

Table 8 shows the categories which obtained a highest bulls-eye score whereas Table 9 shows the results of the 10 categories with the worst bulls-eye score. Both tables have the following structure: (i) name and bulls-eye score of the category; (ii) image of the key shape; (iii) images of the MPEG-7 arranged according to the obtained similarity value.

In Table 8, the most shapes retrieved belong to the same category or they are cognitively similar. For example: (i) the pencil retrieved in the Bottle category have nearly the same shape as any of the bottles; (ii) the device-star retrieved in the Bone category can be built joining the two ends of Bone-12; (iii) the apples retrieved in the Pocket category have the same boundary shape as the clocks; etc. Moreover, analysing the results obtained, it can be deduced that the SimQSD approach is:

i. invariant to rotations, proved by the Hammer, Pencil and Spoon categories;

ii. invariant to translations, proved by the Brick category;

iii. invariant to scaling, proved by the Bone and Pocket categories;

iv. invariant to mirror-image reflections, proved by the Hammer category;

v. influenced by the quantity of relevant points contained by the compared shapes: the more difference in relevant points (or the more relevant points compared to the void point) the lower the similarity.

If both shapes have an approximate number of relevant points, the features of shape of each relevant point (i.e. the edge connection, the angle, the convexity, the type of curvature, etc.) are the ones that influences the similarity more, according to the priorities exemplified in Figure 12. For example, objects retrieved as similar are those whose:

\footnotetext{
${ }^{5}$ http://www.dabi.temple.edu/ shape/MPEG7/results.html
} 
i. shapes have a lot of legs (concavities and convexities) proved by Teddy and Chopper categories;

ii. shapes have round handles or queues proved by Cup and Pocket categories;

iii. shapes have similar curves proved by Heart and Pocket categories;

iv. shapes have similar curves and convexities proved by Comma and Spoon categories.

Note that the $\operatorname{Sim} Q S D$ is intended to calculate a similarity measure between shapes and it has not been designed for object recognition. One aspect to take into account in object retrieval from a database is the similarity of the objects compared, not only the similarity of the shape of its boundary. In order to improve the bulls-eye test score, the $\operatorname{Sim} Q S D$ should be combined with a similarity measure for comparing sizes, for example.

\subsection{Similarity Values Between Tile Images Used to Build Mo- saics}

The SimQSD approach is also used to compare the shapes of tile images captured by an industrial camera AVT-Guppy F033C located on a platform from which a robot arm picks and places tile pieces for building tile mosaics (Figure 14). The closed boundary of the objects within these BMP or JPEG images is extracted using the well-known segmentation method by Canny (1986).

Table 10 shows the similarity values obtained for tile images which were used to build the mosaic in Figure 14. This table shows: (i) image of the tile key shape; (ii) images of the rest of the tiles arranged according to the obtained similarity value. Note that the key shapes are arranged in this table by the number of relevant points. Moreover, as the tile shapes have straight edges with convex relevant points, it is easy to notice the decrease in the similarity value according to the difference in the number of relevant points of both shapes.

Finally, it is also important to realise that the $\operatorname{Sim} Q S D$ approach deals with colour images and that the colour of the objects is not taken into account for calculating the similarity value between their shapes.

\subsection{Similarity Values Between a Collection of Clipart Images}

Clipart images refer to pre-made images composed of illustrations (created by hand or by computer software) which are used commonly in both personal and commercial projects and are easily found on the Web as icons. The Clipart images used in this comparison are BMP images that contain colour or back and white objects. The closed boundary of those objects is extracted by using the colour segmentation method by Felzenszwalb and Huttenlocher (2004). 
Table 11 presents the similarity values obtained for a collection of Clipart images showing: (i) Clipart image with the key shape; and (ii) Clipart images arranged according to the obtained similarity value.

From Table 11, it can be noticed that images which could be considered to belong to the same category have obtained a high similarity value (i.e. the Clipart images of chess pieces or animals), but other images that would be not included in the same category are obtaining high similarity values because they have similar number of relevant points, similar number of curves, similar number of concavities, etc. (i.e. the arrangements obtained for the club, heart and arrow). However, it is important to note that cognitive similarities can be found between the shapes of different objects, as for example: (i) the shape of a card club could be similar to the shape of a cloverleaf, or to the shapes of other leafs, or to the shape of other card symbols such as a spade; (ii) the shape of a heart could be similar to the shape of a leaf, to the shape of a rotated card spade or to the shape of a drop; and (iii) the shape of an arrow could be similar to the shape of a card spade and it is also sharp pointed as a card diamond.

\subsection{Summary of the Results}

After the experimental evaluation, the advantages of our approach are summarized here:

- it provides a similarity value between two shapes but also a set of points with no correspondence in the compared shapes;

- it obtains nearly the same similarity values either using CNDs or interval distances;

- it obtains a similarity value between instances of the same shape that are deformed or incomplete and it also gives an approximate location of such deformation or incompleteness because the relevant points of one shape that do not have a correspondence in the other shape show where the deformation or the incomplete part of the other shape is;

- it obtains a high similarity value between translated, rotated, scaled and symmetrical shapes;

- it can compare colour or black and white images and it does not require any special boundary extraction technique (i.e. Canny (1986), Felzenszwalb and Huttenlocher (2004), etc.).

From a cognitive point of view, the problem of shape equivalence involves understanding the conditions under which people perceive two distinct objects as having the same shape. In addition to the cognitive definition of shape perception 


\section{Z. Falomir et al.}

by Wilson and Keil (1999), Palmer (1989) (see Representing Shape and Structure Chapter) considers that two objects have the same objective shape even after they have undergone spatial transformations such as translations (changing position), rotations (changing orientation), scaling (expanding and contracting in size), mirror-image reflections (changing direction) and combinations of these transformations. However, if spatial transformations such as squashing, stretching or deforming in any way are needed to bring two objects into exact correspondence, then they have different objective shapes, although they can be perceived as very similar. According to this, our approach has proved to fulfill the requirements of a cognitive perception of shape, because it is invariant to translations, rotations and scaling and also obtains a high similarity value between mirror-image reflections or symmetrical shapes.

\section{Conclusions}

A generalization of the qualitative model for shape description (QSD) formulated by Falomir et al. (2008) has been presented in this paper. Then, an approach for calculating a similarity value between two QSDs has been presented (SimQSD). This approach works in three steps: (1) comparing qualitative tags related to the same feature of shape by building dissimilarity matrices using: (a) conceptual neighbourhood diagrams (CNDs); and (b) interval distances; (2) calculating a similarity measure between relevant points; and finally, (3) obtaining a similarity measure between the QSD of the objects by cyclically comparing their relevant points.

Both methods for obtaining dissimilarity matrices for qualitative features of shape (CNDs and interval distances) provide similarity measures that are suitable for our case of study, because there is only a very small difference between them. Furthermore, it is clear that obtaining dissimilarity matrices between qualitative concepts built from CNDs is more intuitive and simpler to calculate. In contrast, dissimilarity matrices between qualitative concepts built from interval distances are more accurate from a mathematical point of view.

The SimQSD approach has been tested using all the images of different categories of the MPEG-7 CE-Shape-1 library, images of tiles used to build mosaics, and a collection of Clipart images. It is proved to be able to compare colour or black and white images and it is independent of the segmentation method used for extracting the boundary of the shape of the object in the image. Moreover, the results obtained show that: (1) the similarity values obtained are invariant to rotations, translations, scaling and mirror-image changes of shapes and also combinations of these; (2) a similarity value can be obtained between deformed or incomplete shapes and the approximate location of the deformation or cut is determined by locating the relevant points with void correspondence; and (3) the 
similarity values obtained by our approach are coherent and cognitive because the lower the difference in shape to the human vision, the higher the similarity.

\section{Acknowledgments}

This research has been partly supported by the project ARTEMISA provided by the Spanish Science and Technology Ministerial Department (TIN2009-14378C02-01), by the Generalitat Valenciana (BFPI06/219, BEFPI/2009/024, BEST/ 2009/148) and by Universitat Jaume I - Fundació Bancaixa (P11A2008-14).

\section{References}

Allen, J. (1981). An Interval-Based Representation of Temporal Knowledge. In Proc. International Joint Conference on Artificial Intelligence (IJCAI), pages 221-226.

Attalla, E. and Siy, P. (2005). Robust shape similarity retrieval based on contour segmentation polygonal multiresolution and elastic matching. Pattern Recognition, 38(12):2229-2241.

Bai, X., Yang, X., and Latecki, L. J. (2008). Detection and recognition of contour parts based on shape similarity. Pattern Recogn., 41(7):2189-2199.

Bay, H., Tuytelaars, T., and Gool, L. V. (2006). SURF: Speed up robust features. In European Conference on Computer Vision (ECCV) Proceedings of, pages $404-417$.

Berretti, S., Bimbo, A. D., and Pala, P. (2000). Retrieval by shape similarity with perceptual distance and effective indexing. IEEE Transactions on Multimedia, 2(4):225-239.

Biederman, I. (1987). Recognition-by-components: A theory of human image understanding. Psychological Review, 94:115-147.

Canny, J. F. (1986). A computational approach to edge detection. IEEE Transactions on Pattern Analysis and Machine Intelligence (TPAMI), 8:679-697.

Clementini, E. and Di Felice, P. (1997). A global framework for qualitative shape description. Geoinformatica, 1(1):11-27.

Cohn, A. (1995). A hierarchical representation of qualitative shape based on connection and convexity. In Proc COSIT95, LNCS, pages 311-326. Springer Verlag. 
Csurka, G., Bray, C., Dance, C., and Fan, L. (2004). Visual categorization with bags of keypoints. In Workshop on Statistical Learning in Computer Vision, European Conference on Computer Vision (ECCV) Proceedings of, pages 1 -22 .

Damski, J. C. and Gero, J. S. (1996). A logic-based framework for shape representation. Computer-aided Design, 28(3):169-181.

Falomir, Z., Almazán, J., Museros, L., and Escrig, M. T. (2008). Describing 2D objects by using qualitative models of color and shape at a fine level of granularity. In Proc. of the Spatial and Temporal Reasoning Workshop at the 23rd AAAI Conference on Artificial Intelligence, ISBN: 978-1-57735-379-9.

Falomir, Z., Jimenez-Ruiz, E., Escrig, M. T., and Museros, L. (2011). Describing images using qualitative models and description logics. Spatial Cognition and Computation, 11:1-30.

Falomir, Z., Martí, I., Viana, W., Museros, L., and Escrig, M. T. (2010). A pragmatic approach for qualitative shape and qualitative colour similarity matching. In Artificial Intelligence Research and Development, Frontiers in Artificial Intelligence and Applications, volume 220, pages 281-290. IOS Press, Amsterdam.

Felzenszwalb, P. F. and Huttenlocher, D. P. (2004). Efficient graph-based image segmentation. International Journal of Computer Vision, 59(2):167-181.

Forsyth, D. A. and Ponce, J. (2003). Computer vision: a modern approach. Upper Saddle River, Prentice Hall.

Freksa, C. (1991). Qualitative spatial reasoning. In Mark, D. M. and Frank, A. U., editors, Cognitive and Linguistic Aspects of Geographic Space, NATO Advanced Studies Institute, pages 361-372. Kluwer, Dordrecht.

Freksa, C. (1992). Using orientation information for qualitative spatial reasoning. In Frank, A. U., Campari, I., and Formentini, U., editors, Theories and Methods of Spatio-Temporal Reasoning in Geographic Space. Intl. Conf. GISFrom Space to Territory, volume 639 of Lecture Notes in Computer Science, pages 162-178, Berlin. Springer.

Galton, A. and Meathrel, R. (1999). Qualitative outline theory. In IJCAI-99, Proceedings of the 16th international joint conference on Artificial intelligence - Volume 2, pages 1061-1066. Morgan Kaufmann Publishers Inc.

Gdalyahu, Y. and Weinshall, D. (1998). Flexible syntactic matching of curves. In Burkhardt, H. and Neumann, B., editors, European Conference on Computer Vision (ECCV (2)), volume 1407 of Lecture Notes in Computer Science, pages 123-139. Springer. 
Gero, J. S. (1999). Representation and reasoning about shapes: Cognitive and computational studies in visual reasoning in design. In Freksa, C. and Mark, D. M., editors, Spatial Information Theory: Cognitive and Computational Foundations of Geographic Information Science, International Conference COSIT, Proceedings, volume 1661 of Lecture Notes in Computer Science (LNCS), pages 315-330. Springer.

González-Abril, L., Cuberos, F., Velasco, F., and Ortega, J. (2009). Ameva: An autonomous discretization algorithm. Expert Systems with Applications, 36(3):5327 - 5332 .

Gonzalez-Abril, L., Velasco, F., Angulo, C., Ortega, J. A., and Ruiz, F. (2004). Sobre núcleos, distancias y similitudes entre intervalos. Inteligencia Artificial. Revista Iberoamericana de IA, 23:111-117.

Gottfried, B. (2008). Qualitative similarity measures: The case of twodimensional outlines. Computer Vision and Image Understanding, 110(1):117-133.

Gottfried, B. (2009). Shape from Positional-contrast Characterising Sketches with Qualitative Line Arrangements. Deutscher Universitätsverlag.

Kuijpers, B., Moelans, B., and de Weghe, N. V. (2006). Qualitative polyline similarity testing with applications to query-by-sketch, indexing and classification. In de By, R. A. and Nittel, S., editors, 14th ACM International Symposium on Geographic Information Systems, ACM-GIS 2006, Proceedings, pages 11-18. ACM.

Latecki, L., Lakamper, R., and Eckhardt, U. (2000). Shape descriptors for nonrigid shapes with a single closed contour. In Proceedings of the Computer Vision on Pattern Recognition (CVPR), pages 424-429.

Latecki, L. J. and Lakamper, R. (2000). Shape similarity measure based on correspondence of visual parts. IEEE Trans. Pattern Analysis and Machine Intelligence, 22(10):1185-1190.

Latecki, L. J., Lu, C., Sobel, M., and Bai, X. (2008). Multiscale random fields with application to contour grouping. In Koller, D., Schuurmans, D., Bengio, Y., and Bottou, L., editors, NIPS, pages 913-920. Curran Associates, Inc.

Lesher, S., Guan, L., and Cohen, A. H. (2000). Symbolic time-series analysis of neural data. Neurocomputing, 32:1073-1081.

Levenshtein, V. (1966). Binary codes capable of correcting deletions, insertions, and reversals. Soviet Physics Doklady, 10(8):707-710. 
Leyton, M. (2004). Shape as memory storage. In Cai, Y., editor, Ambient Intelligence for Scientific Discovery, volume 3345 of Lecture Notes in Computer Science, pages 81-103. Springer.

Lin, J., Keogh, E., Lonardi, S., and Chiu, B. (2003). A symbolic representation of time series, with implications for streaming algorithms. In DMKD '03: Proceedings of the 8th ACM SIGMOD workshop on Research issues in data mining and knowledge discovery, pages 2-11. ACM, New York, NY, USA.

Ling, H. and Jacobs, D. W. (2007). Shape classification using the inner-distance. IEEE Transactions on Pattern Analysis and Machine Intelligence, 29:286299.

Lowe, D. G. (2004). Distinctive image features from scale-invariant keypoints. International Journal of Computer Vision, 60:91-110.

Macrini, D., Siddiqi, K., and Dickinson, S. J. (2008). From skeletons to bone graphs: Medial abstraction for object recognition. In Computer Vision on Pattern Recognition (CVPR). IEEE Computer Society.

Matas, J., Chum, O., Martin, U., and Pajdla, T. (2002). Robust wide baseline stereo from maximally stable extremal regions. In Rosin, P. L. and Marshall, D., editors, Proceedings of the British Machine Vision Conference, BMVA, pages 384-393. London, UK.

Mikolajczyk, K., Tuytelaars, T., Schmid, C., Zisserman, A., Matas, J., Schaffalitzky, F., Kadir, T., and Gool, L. (2005). A comparison of affine region detectors. International Journal of Computer Vision, 65(2):43-72.

Mori, G., Belongie, S., and Malik, J. (2001). Shape contexts enable efficient retrieval of similar shapes. Computer Vision and Pattern Recognition, IEEE Computer Society Conference on, 1:723-730.

Museros, L. (2006). Qualitative theories on shape representation and movement. Application to industrial manufacturing and robotics. $\mathrm{PhD}$ thesis, Departament Ciències de la Computació i Intel.ligència Artificial, Universitat Jaume I, E-12071, Castellón, SPAIN. ISBN: 978-84-691-6583-6 URL: http://www.tdx.cat/TDX-0909108-142621/.

Museros, L. and Escrig, M. T. (2007). Automating assembly of ceramic mosaics using qualitative shape matching. In The 2007 IEEE/RSJ International Conference on Intelligent Robots and Systems, ISBN: 1-4244-0912-8, pages 4096 -4101 .

Museros, L., Falomir, Z., Velasco, F., González-Abril, L., and Martí, I. (2011). 2D qualitative shape matching applied to ceramic mosaic assembling. Journal of Intelligent Manufacturing, 22:1-11. DOI 10.1007/s10845-011-0524-6. 
Palmer, S. (1999). Vision Science: Photons to Phenomenology. The MIT Press.

Palmer, S. E. (1989). Reference frames in the perception of shape and orientation. In Shepp, B. E. and Ballesteros, S., editors, Object perception: Structure and process, pages 121-163. Erlbaum, Hilldale, NJ.

Richards, W. and Hoffman, D. D. (1985). Codon constraints on closed 2D shapes. In Computer Vision, Graphics, and Image Processing, pages 265-281.

Robinson, S. (2007). A statistical process control approach to selecting a warmup period for a discrete-event simulation. European Journal of Operational Research, 176:332-346.

Schlieder, C. (1994). Qualitative shape representation. In $P$ Burrough and $A M$ Frank, editors, Proceedings, GISDATA Specialist Meeting on Geographical Objects with Undetermined Boundaries, pages 123-140. Taylor and Francis.

Schuldt, A., Gottfried, B., and Herzog, O. (2006). Retrieving shapes efficiently by a qualitative shape descriptor: The scope histogram. In Image and Video Retrieval, 5th International Conference, CIVR 2006, Proceedings, pages 261270.

Sebastian, T. B., Klein, P. N., and Kimia, B. B. (2001). Recognition of shapes by editing shock graphs. In In IEEE International Conference on Computer Vision, pages 755-762.

Sebastian, T. B., Klein, P. N., and Kimia, B. B. (2002). Shock-based indexing into large shape databases. In ECCV'02: Proceedings of the 7th European Conference on Computer Vision-Part III, pages 731-746, London, UK. SpringerVerlag.

Shapiro, L., Moriarty, J., Mulgaonkar, P., and Haralick, R. (1980). Sticks, plates, and blobs: Three-dimensional object representation for scene analysis. In Association for the Advancement of Artificial Intelligence (AAAI), pages 2831.

Shokoufandeh, A., Dickinson, S. J., Jönsson, C., Bretzner, L., and Lindeberg, T. (2002). On the representation and matching of qualitative shape at multiple scales. In Heyden, A., Sparr, G., Nielsen, M., and Johansen, P., editors, ECCV (3), volume 2352 of Lecture Notes in Computer Science, pages 759775. Springer.

Siddiqi, K., Shokoufandeh, A., Dickinson, S. J., and Zucker, S. W. (1998). Shock graphs and shape matching. In Proceedings of the Sixth International Conference on Computer Vision (ICCV), page 222, Washington, DC, USA. IEEE Computer Society. 
30 Z. Falomir et al.

Super, B. J. (2004). Fast correspondence-based system for shape retrieval. Pattern Recogn. Lett., 25(2):217-225.

Wilson, R. A. and Keil, F. C., editors (1999). The MIT Encyclopedia of the Cognitive Sciences. The MIT Press, Cambridge, Massachusetts. 


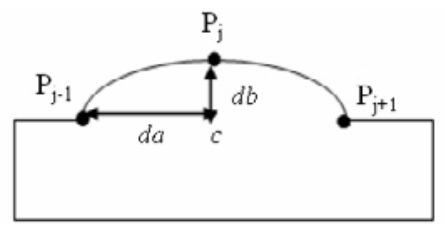

Figure 1: Characterisation of $P_{j}$ a point of curvature.

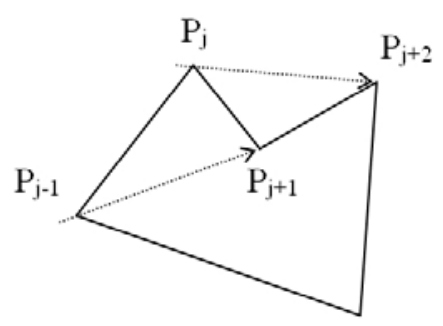

Figure 2: Characterisation of $P_{j}$ as convex and $P_{j+1}$ as concave.

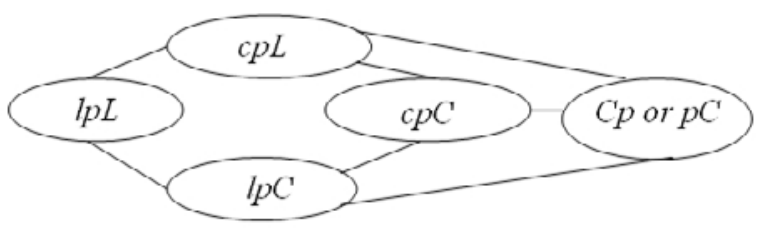

Figure 3: CND for feature Edge Connection (EC). 
32 Z. Falomir et al.

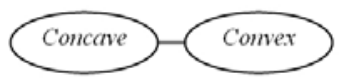

Figure 4: CND for feature Convexity (C).

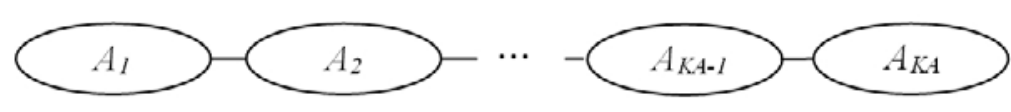

Figure 5: CND for feature Angle (A).

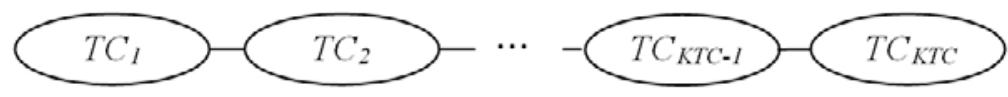

Figure 6: CND for feature Type of Curvature (TC).

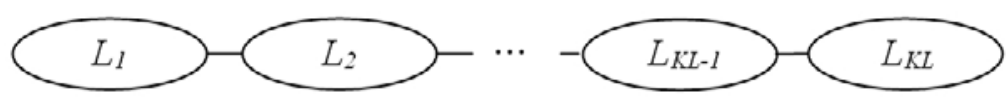

Figure 7: CND for feature compared Length (L).
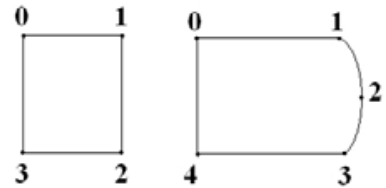

Figure 8: Examples of shapes with different quantities of relevant points.

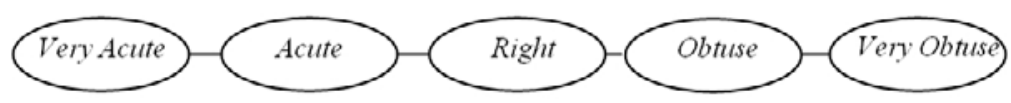

Figure 9: CND for feature Angle (A) determined by experts. 


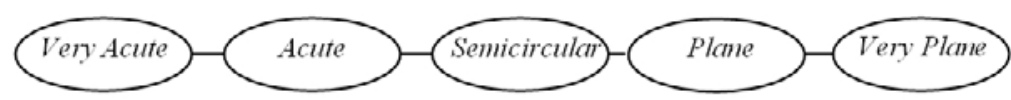

Figure 10: CND for feature Type of Curvature (TC) determined by experts.

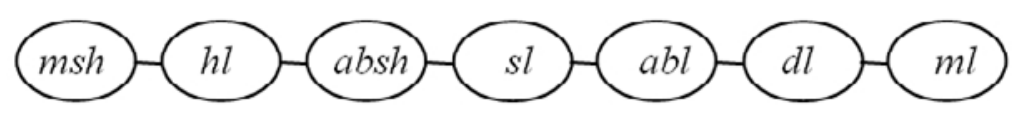

Figure 11: CND for feature compared Length (L) determined by experts: much_shorter (msh), half_length (hl), a_bit_shorter (absh), similar_length (sl), a_bit_longer (abl), double_length $(\mathrm{dl})$, much_longer $(\mathrm{ml})$.
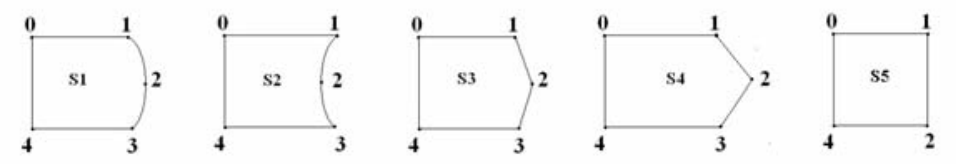

Figure 12: Examples of shapes for explaining the intuitive priorities obtained for C, EC, A, TC and L.

Table 1: Qualitative description of a 2D object containing straight segments and curves.

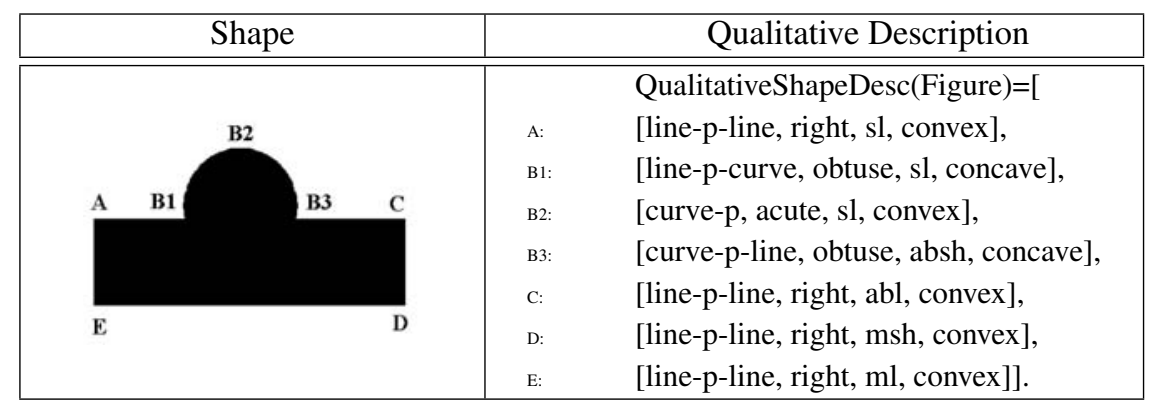




\section{Z. Falomir et al.}

Figure 13: Images from MPEG-7 CE Shape-1 library used for testing our approach. The starting point of the QSD is shown by the number 0 .

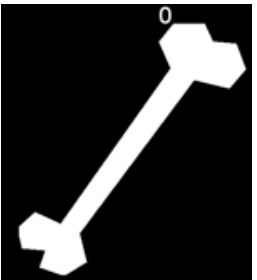

(a) Bone-1

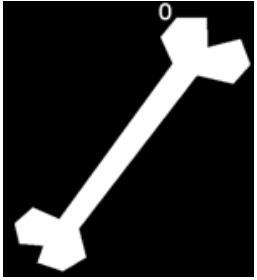

(b) Bone-3

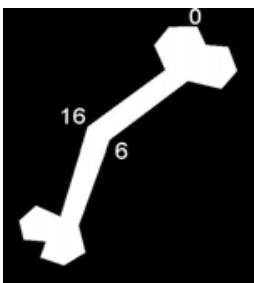

(e) Bone-7

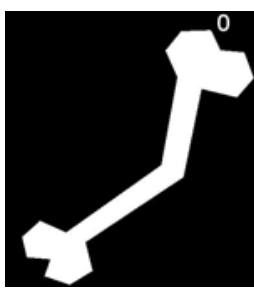

(f) Bone-8

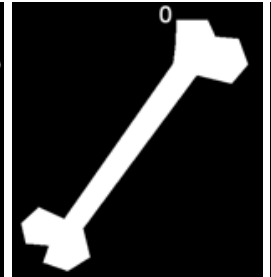

(c) Bone-4

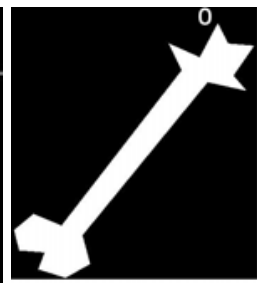

(g) Bone-11

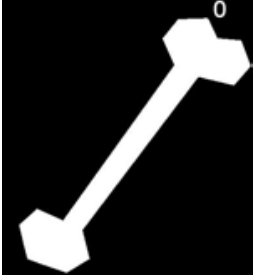

(d) Bone-6

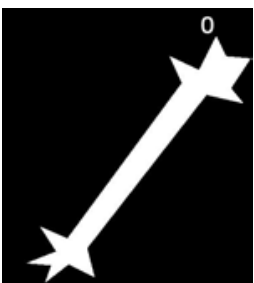

(h) Bone-12

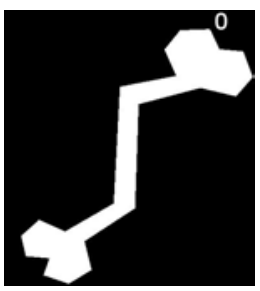

(i) Bone-17

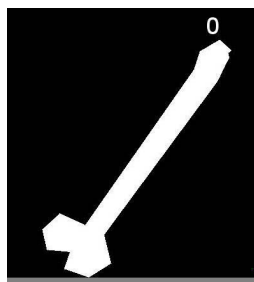

(j) Bone-18

Table 2: Dissimilarity matrix for EC using a CND.

\begin{tabular}{|c|c|c|c|c|c|}
\hline $\boldsymbol{E C}$ & $l p L$ & $l p C$ & $c p L$ & $c p C$ & $c p / p C$ \\
\hline$l p L$ & 0 & 1 & 1 & 2 & 2 \\
\hline$l p C$ & 1 & 0 & 2 & 1 & 1 \\
\hline$c p L$ & 1 & 2 & 0 & 1 & 1 \\
\hline$c p C$ & 2 & 1 & 1 & 0 & 1 \\
\hline$c p / p C$ & 2 & 1 & 1 & 1 & 0 \\
\hline
\end{tabular}


Figure 14: Scenario where automatic assembling of tile mosaics are carried out.

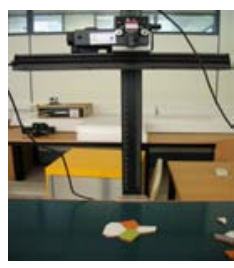

(a) Industrial camera located on a platform

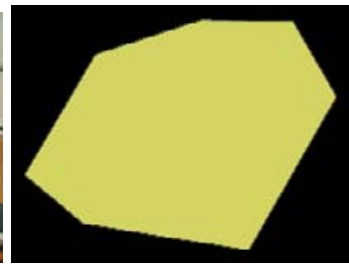

(b) Image obtained

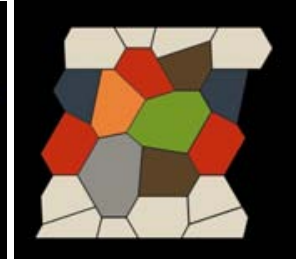

(c) Mosaic to assemble

Table 3: Dissimilarity matrix for $\mathbf{C}$ using a CND.

\begin{tabular}{|c|c|c|}
\hline Convexity & Concave & Convex \\
\hline Concave & 0 & 1 \\
\hline Convex & 1 & 0 \\
\hline
\end{tabular}

Table 4: Distance matrix for TC and A using interval distances.

\begin{tabular}{|c|c|c|c|c|c|}
\hline $\begin{array}{c}\text { TC } \\
\text { or } \boldsymbol{A}\end{array}$ & $\begin{array}{c}\text { Very } \\
\text { acute }\end{array}$ & Acute & $\begin{array}{c}\text { Semi- } \\
\text { circular } \\
\text { or Right }\end{array}$ & $\begin{array}{c}\text { Plane or } \\
\text { Obtuse }\end{array}$ & $\begin{array}{c}\text { Very } \\
\text { plane } \\
\text { or Very } \\
\text { obtuse }\end{array}$ \\
\hline Very acute & 0.0 & 42.6 & 71.6 & 97.5 & 140.0 \\
\hline Acute & 42.6 & 0.0 & 32.6 & 55.0 & 97.5 \\
\hline $\begin{array}{c}\text { Semi- } \\
\text { circular } \\
\text { or Right }\end{array}$ & 71.6 & 32.6 & 0.0 & 32.6 & 71.6 \\
\hline $\begin{array}{c}\text { Plane or } \\
\text { Obtuse }\end{array}$ & 97.5 & 55.0 & 32.6 & 0.0 & 42.6 \\
\hline $\begin{array}{c}\text { Very } \\
\text { plane } \\
\text { or Very } \\
\text { obtuse }\end{array}$ & 140.0 & 97.5 & 71.6 & 42.6 & 0.0 \\
\hline
\end{tabular}


36 Z. Falomir et al.

Table 5: Distance matrix for qualitative compared length using interval distances.

\begin{tabular}{|c|c|c|c|c|c|c|c|}
\hline Length & $\mathrm{msh}$ & $\mathrm{hl}$ & $\mathrm{qsh}$ & $\mathrm{sl}$ & $\mathrm{ql}$ & $\mathrm{dl}$ & $\mathrm{ml}$ \\
\hline$m s h$ & 0.00 & 0.32 & 0.55 & 0.81 & 1.32 & 1.80 & 6.95 \\
\hline$h l$ & 0.32 & 0.00 & 0.25 & 0.50 & 1.04 & 1.50 & 6.75 \\
\hline$q s h$ & 0.55 & 0.25 & 0.00 & 0.25 & 0.79 & 1.25 & 6.52 \\
\hline$s l$ & 0.81 & 0.50 & 0.25 & 0.00 & 0.58 & 1.00 & 6.35 \\
\hline$q l$ & 1.32 & 1.04 & 0.79 & 0.58 & 0.00 & 0.58 & 5.77 \\
\hline$d l$ & 1.80 & 1.50 & 1.25 & 1.00 & 0.58 & 0.00 & 5.59 \\
\hline $\boldsymbol{m l}$ & 6.95 & 6.75 & 6.52 & 6.35 & 5.77 & 5.59 & 0.00 \\
\hline
\end{tabular}


Similarities Between Qualitative Shape Descriptions

Table 6: SimQSD built from CNDs tested on some Bone shapes of MPEG-7.

\begin{tabular}{|c|c|c|c|c|c|c|c|c|c|}
\hline Bone & 3 & 4 & 6 & 7 & 8 & 11 & 12 & 17 & 18 \\
\hline 1 & $\begin{array}{c}0.98 \\
(0,0) \\
\emptyset\end{array}$ & $\begin{array}{l}0.93 \\
(0,1) \\
\{0\}\end{array}$ & $\begin{array}{c}0.88 \\
(0,15) \\
\{10,11\}\end{array}$ & $\begin{array}{c}0.88 \\
(1,0) \\
\{6,16\}\end{array}$ & $\begin{array}{c}0.88 \\
(1,0) \\
\{6,16\}\end{array}$ & $\begin{array}{c}\mathbf{0 . 9 3} \\
(0,17) \\
\emptyset\end{array}$ & $\begin{array}{c}\mathbf{0 . 8 6} \\
(0,17) \\
\emptyset\end{array}$ & $\begin{array}{c}0.79 \\
(1,0) \\
\{5,7 \\
16,18\}\end{array}$ & $\begin{array}{c}0.64 \\
(0,0) \\
\{0,1,2 \\
3,6,16\}\end{array}$ \\
\hline 3 & 1 & $\begin{array}{l}0.92 \\
(0,1) \\
\{1\}\end{array}$ & $\begin{array}{l}0.88 \\
(0,8) \\
\{1,2\}\end{array}$ & $\begin{array}{c}0.88 \\
(1,0) \\
\{6,16\}\end{array}$ & $\begin{array}{c}0.87 \\
(1,0) \\
\{6,16\}\end{array}$ & $\begin{array}{c}\mathbf{0 . 9 2} \\
(0,17) \\
\emptyset\end{array}$ & $\begin{array}{c}\mathbf{0 . 8 5} \\
(0,17) \\
\emptyset\end{array}$ & $\begin{array}{c}0.79 \\
(1,0) \\
\{5,7 \\
16,18\}\end{array}$ & $\begin{array}{c}0.64 \\
(0,0) \\
\{0,2,3 \\
4,6,16\}\end{array}$ \\
\hline 4 & & 1 & $\begin{array}{c}0.91 \\
(0,7) \\
\{2\}\end{array}$ & $\begin{array}{c}0.82 \\
(2,0) \\
\{0,6,16\}\end{array}$ & $\begin{array}{c}0.82 \\
(2,0) \\
\{0,6,16\}\end{array}$ & $\begin{array}{l}0.89 \\
(1,0) \\
\{17\}\end{array}$ & $\begin{array}{l}\mathbf{0 . 8 2} \\
(1,0) \\
\{17\}\end{array}$ & $\begin{array}{c}0.74 \\
(2,0) \\
\{0,5,7, \\
16,18\}\end{array}$ & $\begin{array}{c}0.68 \\
(0,11) \\
\{0,2,3, \\
6,16\}\end{array}$ \\
\hline 6 & & & 1 & $\begin{array}{c}0.79 \\
(0,0) \\
\{6,10 \\
11,16\}\end{array}$ & $\begin{array}{c}0.78 \\
(9,0) \\
\{1,2 \\
6,16\}\end{array}$ & $\begin{array}{c}\mathbf{0 . 8 1} \\
(8,0) \\
\{1,17\}\end{array}$ & $\begin{array}{c}\mathbf{0 . 7 6} \\
(0,0) \\
\{10,12\}\end{array}$ & $\begin{array}{c}0.70 \\
(0,0) \\
\{5,7, \\
12,15, \\
16,18\}\end{array}$ & $\begin{array}{c}0.71 \\
(0,5) \\
\{6,8 \\
10,12\}\end{array}$ \\
\hline 7 & & & & 1 & $\begin{array}{c}0.97 \\
(0,10) \\
\emptyset\end{array}$ & $\begin{array}{c}\mathbf{0 . 8 3} \\
(0,0) \\
\{6,16\}\end{array}$ & $\begin{array}{c}\mathbf{0 . 7 7} \\
(0,0) \\
\{6,16\}\end{array}$ & $\begin{array}{c}0.86 \\
(0,0) \\
\{7,16\}\end{array}$ & $\begin{array}{c}0.58 \\
(0,1) \\
\{0,1,2, \\
3,5,6, \\
17,18\}\end{array}$ \\
\hline 8 & & & & & 1 & $\begin{array}{c}\mathbf{0 . 8 3} \\
(0,0) \\
\{6,16\}\end{array}$ & $\begin{array}{c}\mathbf{0 . 7 7} \\
(0,0) \\
\{6,16\}\end{array}$ & $\begin{array}{c}0.87 \\
(0,0) \\
\{5,18\}\end{array}$ & $\begin{array}{c}0.58 \\
(0,0) \\
\{0,1,2, \\
3,5,6, \\
16,18\}\end{array}$ \\
\hline 11 & & & & & & 1 & $\begin{array}{c}\mathbf{0 . 9 3} \\
(0,0) \\
\emptyset\end{array}$ & $\begin{array}{c}\mathbf{0 . 7 4} \\
(0,1) \\
\{0,5,7, \\
21\}\end{array}$ & $\begin{array}{c}0.64 \\
(0,1) \\
\{0,1,2 \\
3,4,17\}\end{array}$ \\
\hline 12 & & & & & & & 1 & $\begin{array}{c}\mathbf{0 . 6 9} \\
(1,0) \\
\{0,5,7 \\
21\}\end{array}$ & $\begin{array}{c}\mathbf{0 . 5 7} \\
(0,1) \\
\{1,3,5 \\
12,14 \\
15\}\end{array}$ \\
\hline 17 & & & & 3 & & & & 1 & $\begin{array}{l}0.52 \\
(0,10) \\
\{0,1,2,3, \\
5,6,7,16, \\
19,20\}\end{array}$ \\
\hline
\end{tabular}




\section{Z. Falomir et al.}

Table 7: SimQSD tested on some shapes using dissimilarity matrices built from interval distances for the features of $\mathrm{A}, \mathrm{TC}$ and $\mathrm{L}$.

\begin{tabular}{|c|c|c|c|c|c|c|c|c|c|}
\hline Bone & 3 & 4 & 6 & 7 & 8 & 11 & 12 & 17 & 18 \\
\hline 1 & $\begin{array}{c}\mathbf{1 . 0 0} \\
(0,0) \\
\emptyset\end{array}$ & $\begin{array}{l}0.94 \\
(0,1) \\
\{0\}\end{array}$ & $\begin{array}{c}0.89 \\
(0,8) \\
\{1,2\}\end{array}$ & $\begin{array}{c}0.90 \\
(1,0) \\
\{6,16\}\end{array}$ & $\begin{array}{c}0.90 \\
(1,0) \\
\{6,16\}\end{array}$ & $\begin{array}{c}\mathbf{0 . 9 7} \\
(0,17) \\
\emptyset\end{array}$ & $\begin{array}{c}\mathbf{0 . 9 4} \\
(0,17) \\
\emptyset\end{array}$ & $\begin{array}{c}0.80 \\
(1,0) \\
\{5,7 \\
16,18\}\end{array}$ & $\begin{array}{c}0.65 \\
(0,0) \\
\{1,2,4, \\
5,6,16\}\end{array}$ \\
\hline 3 & 1 & $\begin{array}{l}0.94 \\
(0,1) \\
\{1\}\end{array}$ & $\begin{array}{l}0.89 \\
(0,8) \\
\{0,2\}\end{array}$ & $\begin{array}{c}0.90 \\
(1,0) \\
\{6,16\}\end{array}$ & $\begin{array}{c}0.90 \\
(1,0) \\
\{6,16\}\end{array}$ & $\begin{array}{c}\mathbf{0 . 9 6} \\
(0,17) \\
\emptyset\end{array}$ & $\begin{array}{c}\mathbf{0 . 9 4} \\
(0,17) \\
\emptyset\end{array}$ & $\begin{array}{c}0.80 \\
(1,0) \\
\{5,7 \\
17,18\}\end{array}$ & $\begin{array}{c}0.65 \\
(0,0) \\
\{0,1,2, \\
4,6,16\}\end{array}$ \\
\hline 4 & & 1 & $\begin{array}{c}0.93 \\
(0,7) \\
\{2\}\end{array}$ & $\begin{array}{l}0.85 \\
(10,0) \\
\{6,9,16\}\end{array}$ & $\begin{array}{c}0.85 \\
(2,0) \\
\{0,6,16\}\end{array}$ & $\begin{array}{l}0.92 \\
(1,0) \\
\{17\}\end{array}$ & $\begin{array}{l}\mathbf{0 . 9 0} \\
(1,0) \\
\{17\}\end{array}$ & $\begin{array}{c}0.75 \\
(1,0) \\
\{5,7,10, \\
16,18\}\end{array}$ & $\begin{array}{c}0.69 \\
(0,11) \\
\{0,2,3 \\
6,16\}\end{array}$ \\
\hline 6 & & & 1 & $\begin{array}{l}0.80 \\
(9,0) \\
\{1,2 \\
6,16\}\end{array}$ & $\begin{array}{l}0.80 \\
(9,0) \\
\{1,2, \\
6,16\}\end{array}$ & $\begin{array}{l}\mathbf{0 . 8 7} \\
(9,0) \\
\{1,3\}\end{array}$ & $\begin{array}{l}\mathbf{0 . 8 4} \\
(9,0) \\
\{1,3\}\end{array}$ & $\begin{array}{c}0.70 \\
(9,0) \\
\{1,2 \\
5,7 \\
16,18\}\end{array}$ & $\begin{array}{c}0.73 \\
(0,5) \\
\{6,8 \\
10,12\}\end{array}$ \\
\hline 7 & & & & 1 & $\begin{array}{c}\mathbf{0 . 9 9} \\
(0,10) \\
\emptyset\end{array}$ & $\begin{array}{c}\mathbf{0 . 8 7} \\
(0,0) \\
\{6,16\}\end{array}$ & $\begin{array}{c}\mathbf{0 . 8 4} \\
(0,0) \\
\{6,16\}\end{array}$ & $\begin{array}{c}0.88 \\
(10,0) \\
\{5,18\}\end{array}$ & $\begin{array}{c}0.59 \\
(0,1) \\
\{0,1,2 \\
3,5,6 \\
16,17\}\end{array}$ \\
\hline 8 & & & & & 1 & $\begin{array}{c}\mathbf{0 . 8 7} \\
(0,0) \\
\{6,16\}\end{array}$ & $\begin{array}{c}\mathbf{0 . 8 4} \\
(0,0) \\
\{6,16\}\end{array}$ & $\begin{array}{c}0.89 \\
(0,0) \\
\{5,18\}\end{array}$ & $\begin{array}{c}0.59 \\
(0,0) \\
\{0,1,2, \\
5,16,17, \\
18,19\}\end{array}$ \\
\hline 11 & & & & & & 1 & $\begin{array}{c}\mathbf{0 . 9 7} \\
(0,0) \\
\emptyset\end{array}$ & $\begin{array}{c}0.78 \\
(0,9) \\
\{9,10 \\
16,18\}\end{array}$ & $\begin{array}{c}0.65 \\
(0,11) \\
\{1,3,5 \\
15,16 \\
17\}\end{array}$ \\
\hline 12 & & & & & & & 1 & $\begin{array}{c}\mathbf{0 . 7 6} \\
(0,0) \\
\{9,10 \\
16,18\}\end{array}$ & $\begin{array}{c}\mathbf{0 . 6 4} \\
(0,11) \\
\{1,3,5 \\
12,14 \\
15\}\end{array}$ \\
\hline 17 & & & & 38 & & & & 1 & $\begin{array}{c}0.53 \\
(0,1) \\
\{0,1,2,3, \\
5,6,7,16, \\
19,20\}\end{array}$ \\
\hline
\end{tabular}


Table 8: The Top 10 results of testing $\operatorname{Sim} Q S D$ on all the categories from MPEG-7 Shape Library using CNDs and interval distances.

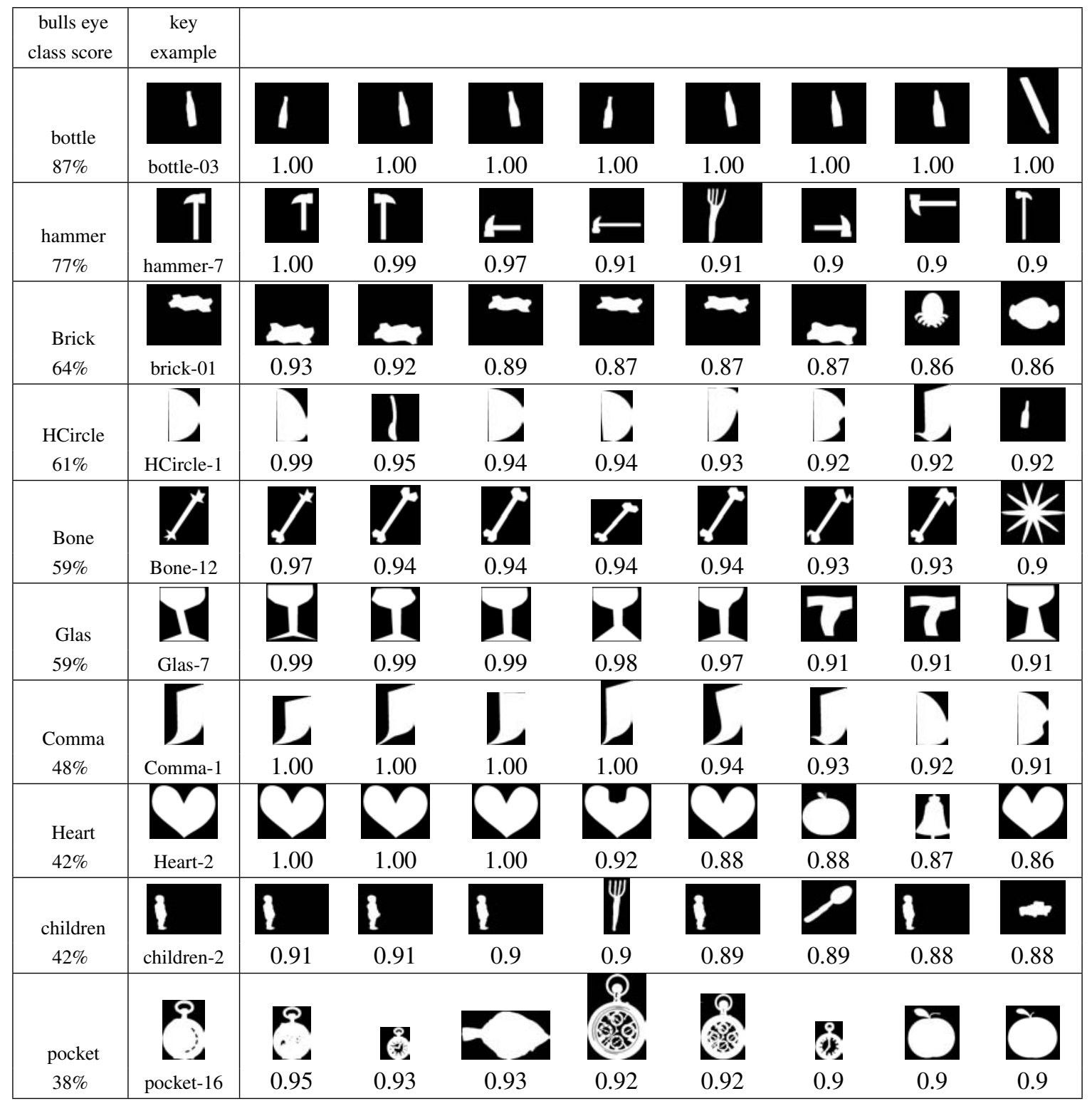


Table 9: The Bottom 10 results of testing SimQSD on all the categories from MPEG-7 Shape Library using CNDs and interval distances.

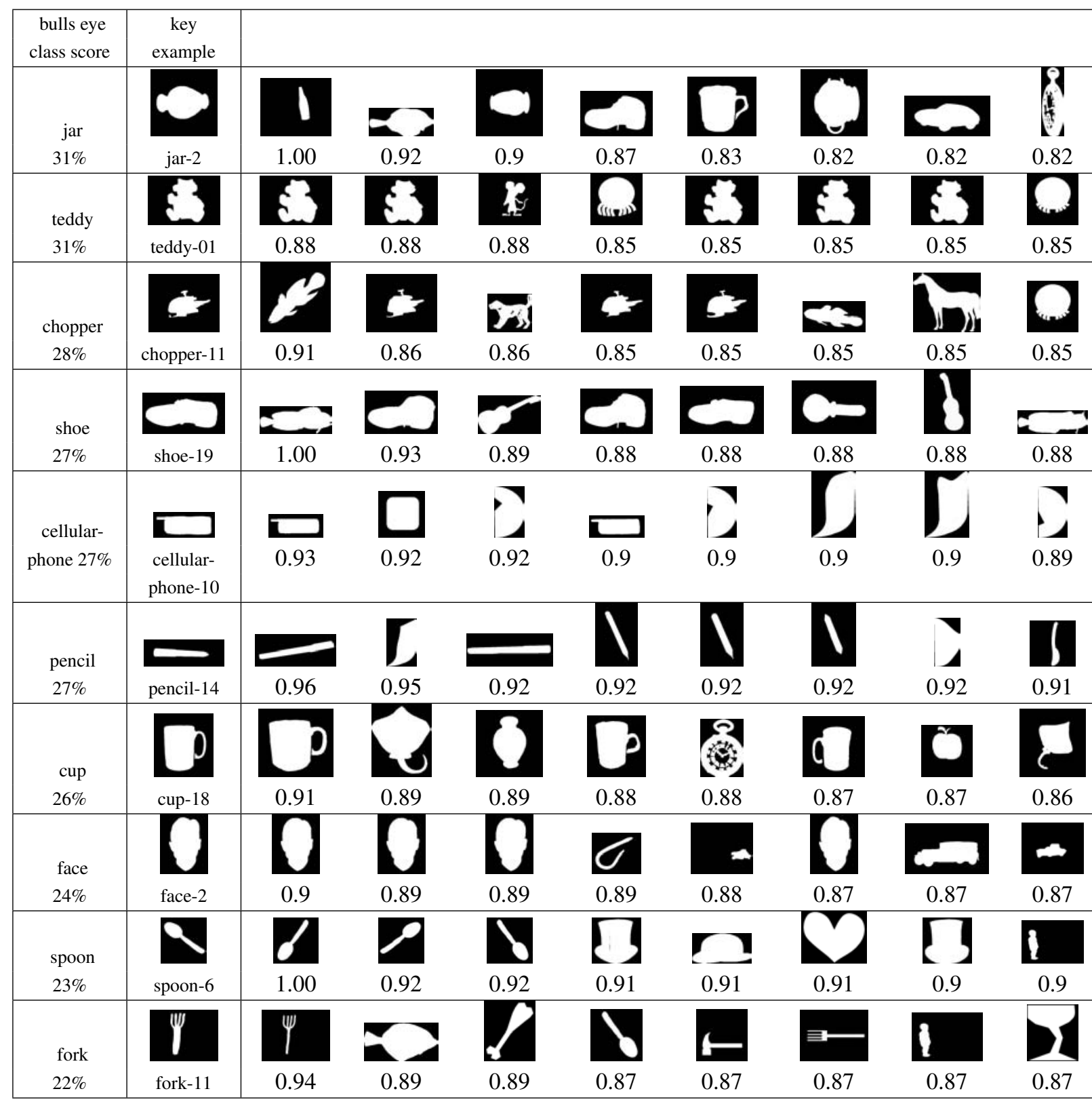


Table 10: Results of testing $\operatorname{Sim} Q S D$ on tile images used to build mosaics.

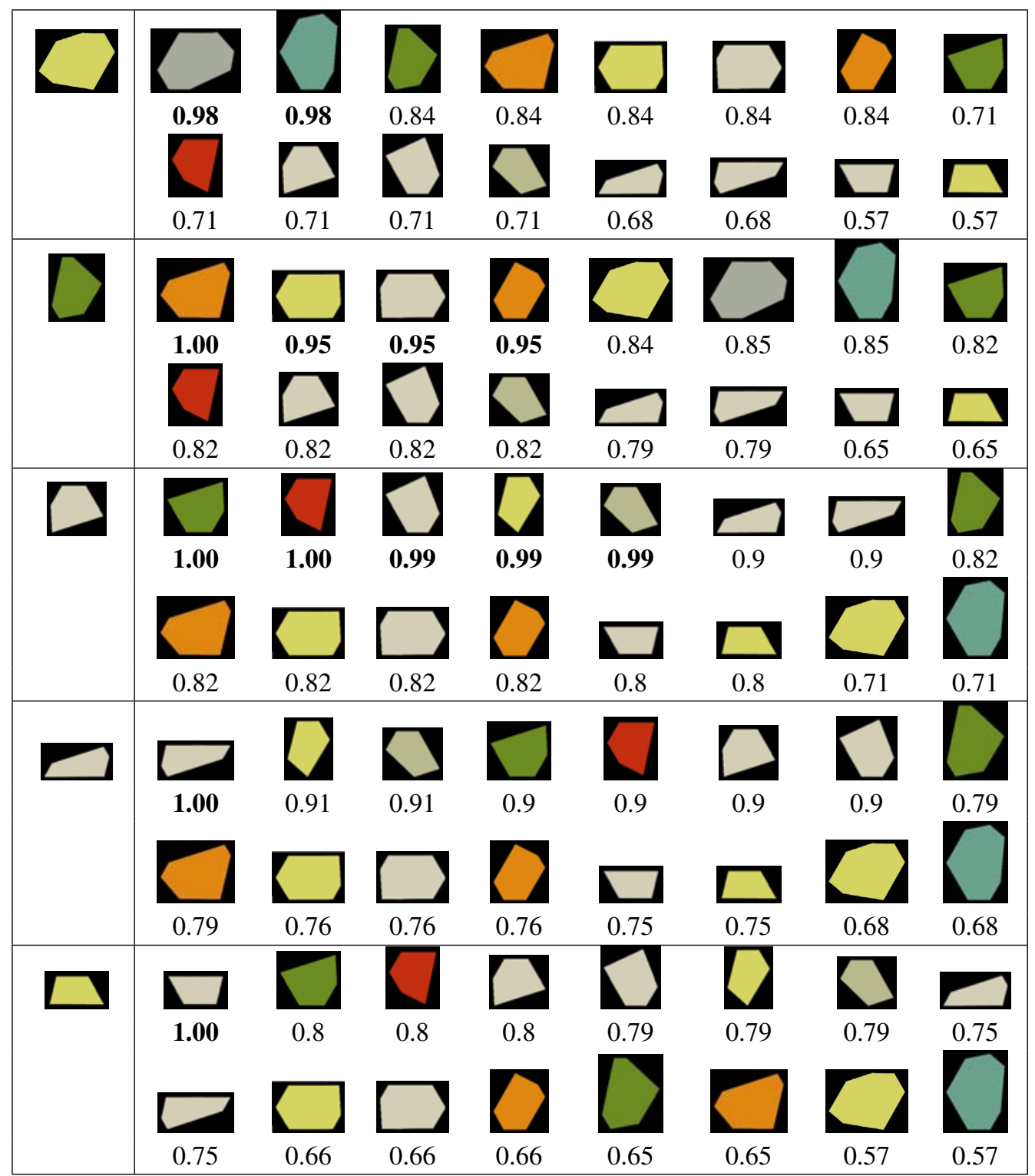


Table 11: Results of testing SimQSD on some Clipart images.

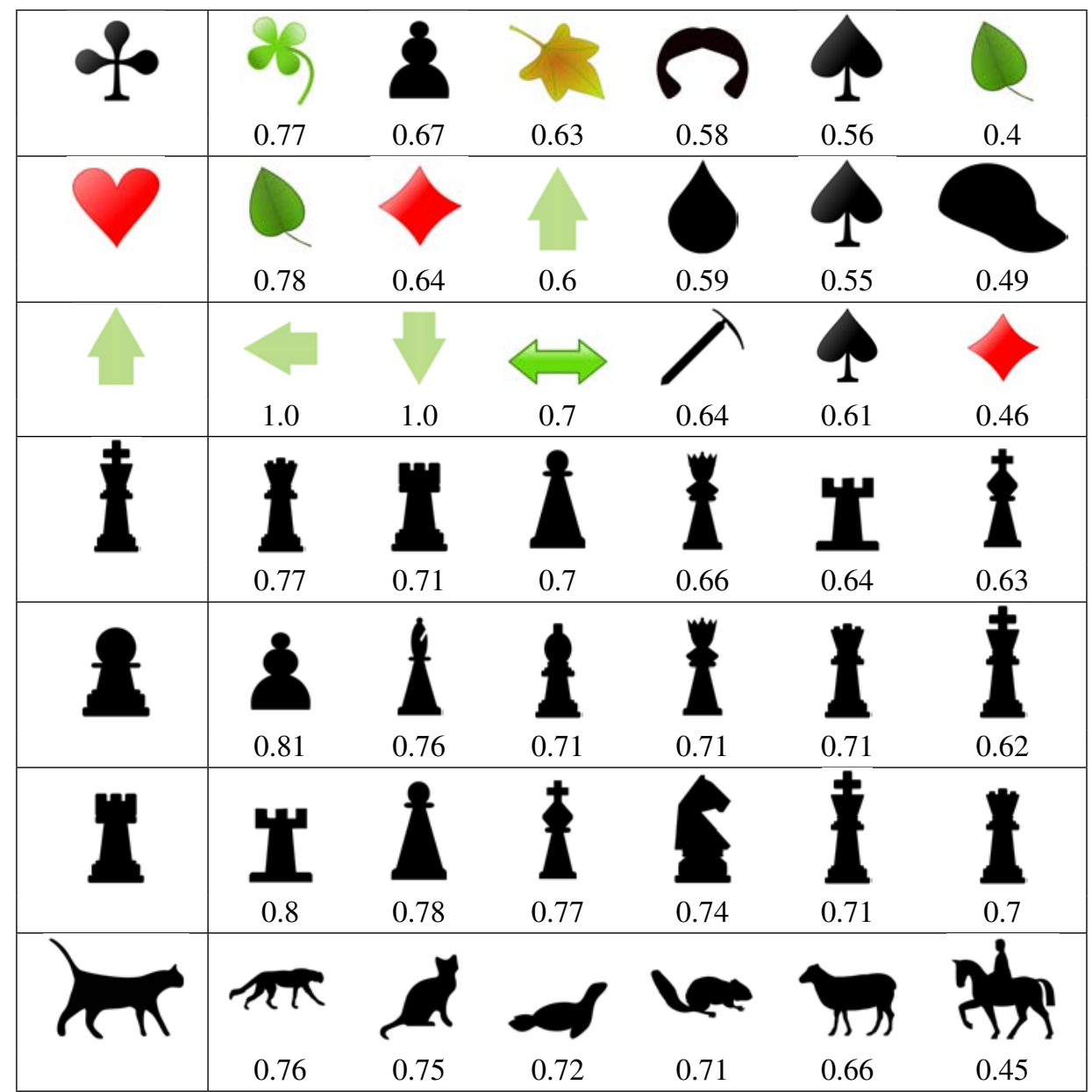

